\title{
Biosynthesis and heterologous production of mycosporine-like amino acids palythines
}

\author{
Manyun Chen, Garret M. Rubin, Guangde Jiang, Zachary Raad, Yousong Ding* \\ Department of Medicinal Chemistry, Center for Natural Products, Drug Discovery and \\ Development (CNPD3), University of Florida, Gainesville, Florida, 32610, United States
}




\section{Table of Contents:}

Table S1. Accession numbers for MysC homologs shown in Figure 2A.............................. S3

Table S2. Bioinformatic analysis of the MAA gene cluster from Nostoc linkia NIES-25.............S5

Table S3. Comparison of ${ }^{1} \mathrm{H}$ and ${ }^{13} \mathrm{C}\{1 \mathrm{H}\}$ NMR chemical shifts of palythine-Thr determined in the

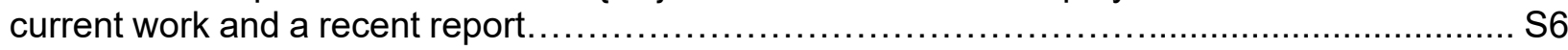

Table S4. Primers used to construct refactored BGCs and express MysD............................. S6

Figure S1. Sequence similarity network (SSN) analysis of protein family \#02655....................S7

Figure S2. Sequence alignment of all phytanoyl-CoA dioxygenases....................................S8

Figure S3. TIC and EIC traces of methanolic extracts (A) and HRMS and MS/MS analysis (B)...S9

Figure S4. The maximal UV absorbance and HRMS spectra of 4-DG (A) and MG (B)............S10

Figure S5. The maximal UV absorbance, HRMS and MS/MS spectra of porphyra-334 ............S11

Figure S6. The maximal UV absorbance, HRMS and MS/MS spectra of Mg-Ala.....................S12

Figure S7. The maximal UV absorbance, HRMS and MS/MS spectra of shinorine................S13

Figure S8. HPLC trace of methanolic extract of $E$. coli expressing mys $A B C D H$ (bottom) and

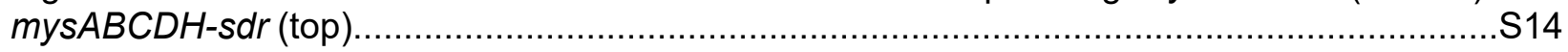

Figure S9. The maximal UV absorbance, HRMS and MS/MS spectra of palythine-Thr ............S14

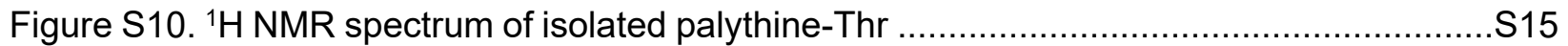

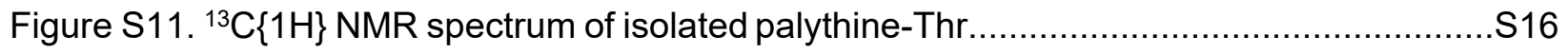

Figure S12. 2D NMR spectra of isolated palythine-threonine ............................................. 18

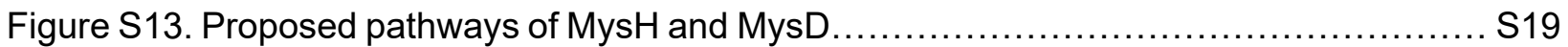

Figure S14. The maximal UV absorbance, HRMS and MS/MS spectra of palythine-Ser .......S20

Figure S15. The maximal UV absorbance, HRMS and MS/MS spectra of palythine-Ala .......S21

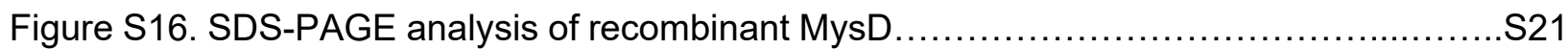

Figure S17. Determination of optimal temperature and $\mathrm{pH}$ for the MysD reaction..............S22

Figure S18. HPLC trace of the MysD reactions with MG and all 20 amino acids as substrates...S23

Figure S19. The maximal UV absorbance, HRMS and MS/MS spectra of MG-Arg.................S24

Figure S20. The maximal UV absorbance, HRMS and MS/MS spectra of MG-Cys................S25

Figure S21. The maximal UV absorbance, HRMS and MS/MS spectra of mycosporine-2-Gly...S26

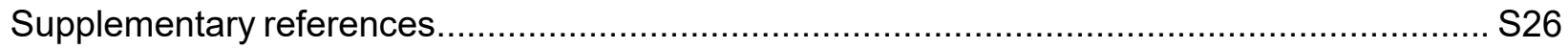


Table S1. Accession numbers for MysC homologs shown in Figure 2A.

\begin{tabular}{|c|c|c|c|}
\hline Uniprot ID & Gene Name & Species & Phylum \\
\hline A0A0Q2QHP0 & AO501_14480 & Mycobacterium gordonae & Actinobacteria \\
\hline A0A3S0TU06 & EKK34_29475 & Mycobacterium sp. & Actinobacteria \\
\hline A0A5A7SAT3 & FOY51_14930 & Rhodococcus sp. C1-24 & Actinobacteria \\
\hline A0A0G4HZ53 & Cvel_9647 & Chromera velia CCMP2878 & Chromerida \\
\hline R1G4T9 & EMIHUDRAFT_52960 & Emiliania huxleyi & Haptista \\
\hline A0A433W0B3 & DSM107010_29350 & Chroococcidiopsis cubana SAG 39.79 & Cyanobacteria \\
\hline A0A139WZN8 & WA1_05090 & Scytonema hofmannii PCC 7110 & Cyanobacteria \\
\hline A0A2Z5X784 & mysC & Nostoc verrucosum & Cyanobacteria \\
\hline A0A1Z4GTP3 & NIES2100_06370 & Calothrix sp. NIES-2100 & Cyanobacteria \\
\hline A0A1Q4RU46 & NIES2101_15200 & Calothrix sp. HK-06 & Cyanobacteria \\
\hline A0A0C2R3C6 & SD80_01670 & Scytonema tolypothrichoides VB-61278 & Cyanobacteria \\
\hline A0A2R5FKA4 & NIES4072_28690 & Nostoc commune NIES-4072 & Cyanobacteria \\
\hline AOAOMOSH70 & AMR41_24200 & Hapalosiphon sp. MRB220 & Cyanobacteria \\
\hline A0A2T1F866 & C7B80_31420 & $\begin{array}{l}\text { Cyanosarcina cf. burmensis CCALA } \\
770\end{array}$ & Cyanobacteria \\
\hline A0A367QNV7 & A6S26_15830 & Nostoc sp. ATCC 43529 & Cyanobacteria \\
\hline A0A2N6JWS5 & CEN44_24325 & Fischerella muscicola CCMEE 5323 & Cyanobacteria \\
\hline B4VP63 & MC7420_4633 & $\begin{array}{l}\text { Coleofasciculus chthonoplastes PCC } \\
7420\end{array}$ & Cyanobacteria \\
\hline K9QUQ5 & Nos7524_3368 & Nostoc sp. PCC 7524 & Cyanobacteria \\
\hline A0A0S3U2V2 & LEP3755_23100 & Leptolyngbya sp. NIES-3755 & Cyanobacteria \\
\hline K9TVZ3 & Chro_0780 & Chroococcidiopsis thermalis PCC 7203 & Cyanobacteria \\
\hline A0A2N6MZD6 & CEN39_11340 & Fischerella thermalis CCMEE 5201 & Cyanobacteria \\
\hline A0A218PXL8 & NIES3585_03720 & Nodularia sp. NIES-3585 & Cyanobacteria \\
\hline A0A1Z4HW63 & NIES2107_59490 & Nostoc carneum NIES-2107 & Cyanobacteria \\
\hline A0A1Z4LYV8 & NIES267_58470 & Calothrix parasitica NIES-267 & Cyanobacteria \\
\hline A0A654SJH1 & apha_01438 & Chrysosporum ovalisporum & Cyanobacteria \\
\hline A0A2C6VZE1 & VF13_24910 & Nostoc linckia z16 & Cyanobacteria \\
\hline A0A2T1EQS1 & C7B70_02210 & Chlorogloea sp. CCALA 695 & Cyanobacteria \\
\hline A0A1E5QWM1 & A5482_11085 & Cyanobacterium sp. IPPAS B-1200 & Cyanobacteria \\
\hline A0A2I8ACV8 & CLI64_23890 & Nostoc sp. CENA543 & Cyanobacteria \\
\hline A0A2D3HK59 & mylE & Nostoc flagelliforme & Cyanobacteria \\
\hline A0A367QJH7 & A6V25_22315 & Nostoc sp. ATCC 53789 & Cyanobacteria \\
\hline A0A2S6VI18 & B1A85_06375 & Chroococcidiopsis sp. TS-821 & Cyanobacteria \\
\hline K9X913 & Glo7428_0523 & Gloeocapsa sp. PCC 7428 & Cyanobacteria \\
\hline A0A1Y0RL91 & BZZ01_16725 & Nostocales cyanobacterium HT-58-2 & Cyanobacteria \\
\hline A0A2P8QMI8 & C7Y66_19855 & Chroococcidiopsis sp. CCALA 051 & Cyanobacteria \\
\hline A0A6B3P645 & F6K60_05300 & Okeania sp. SIO1F9 & Cyanobacteria \\
\hline A0A6B3MZW3 & F6J89_01825 & Symploca sp. SIO1C4 & Cyanobacteria \\
\hline A0A2K8WS68 & AA637_12615 & Cyanobacterium sp. HL-69 & Cyanobacteria \\
\hline A0A4Q9JE38 & B4U84_12935 & Westiellopsis prolifica IICB1 & Cyanobacteria \\
\hline Q3M6C5 & Ava_3856 & Anabaena variabilis ATCC 29413 & Cyanobacteria \\
\hline
\end{tabular}




\begin{tabular}{|c|c|c|c|}
\hline A0A252E4S5 & BV372_13530 & Nostoc sp. T09 & Cyanobacteria \\
\hline A0A367RKS4 & A6770_15820 & Nostoc minutum NIES-26 & Cyanobacteria \\
\hline A0A1E2WNZ8 & A4S05 34795 & Nostoc sp. KVJ20 & Cyanobacteria \\
\hline A0A1B2CWG9 & UCFS15_00407 & Heteroscytonema crispum UCFS15 & Cyanobacteria \\
\hline A0A1U7HY56 & NIES1031_04760 & Chroogloeocystis siderophila 5.2 s.c. 1 & Cyanobacteria \\
\hline A0A1L9QXK4 & BI308_00105 & Roseofilum reptotaenium AO1-A & Cyanobacteria \\
\hline A0A2L2NR98 & NLP_2817 & $\begin{array}{l}\text { Nostoc sp. 'Lobaria pulmonaria (5183) } \\
\text { cyanobiont' }\end{array}$ & Cyanobacteria \\
\hline A0A2H2XFD9 & NIES4071_48500 & Calothrix sp. NIES-4071 & Cyanobacteria \\
\hline A0A533NZW2 & EBE86_16905 & Hormoscilla sp. GUM202 & Cyanobacteria \\
\hline A0A367RVN3 & A6769_04950 & Nostoc punctiforme NIES-2108 & Cyanobacteria \\
\hline A0A1Z4TPY4 & NIES4106_37630 & Fischerella sp. NIES-4106 & Cyanobacteria \\
\hline A0A6B3MAD2 & F6K58_17255 & Symploca sp. SIO2E9 & Cyanobacteria \\
\hline A0A1Z4IH51 & NIES2111_57410 & Nostoc sp. NIES-2111 & Cyanobacteria \\
\hline A0A1Z4IB36 & NIES2111_35850 & Nostoc sp. NIES-2111 & Cyanobacteria \\
\hline K9VKW1 & Osc7112_3784 & Oscillatoria nigro-viridis PCC 7112 & Cyanobacteria \\
\hline A0A2T1F5R3 & C7B77_28500 & $\begin{array}{l}\text { Chamaesiphon polymorphus CCALA } \\
037\end{array}$ & Cyanobacteria \\
\hline K9W0D3 & Cri9333_2377 & Crinalium epipsammum PCC 9333 & Cyanobacteria \\
\hline A0A1Z4SWP6 & NIES4105_48440 & Calothrix sp. NIES-4105 & Cyanobacteria \\
\hline A0A1U7I932 & FACHB389_18875 & Nostoc calcicola FACHB-389 & Cyanobacteria \\
\hline A0A1W5CLX0 & AN489_06955 & Anabaena sp. 39858 & Cyanobacteria \\
\hline A0A328IAQ4 & C6Y22_26065 & $\begin{array}{l}\text { Hapalosiphonaceae cyanobacterium } \\
\text { JJU2 }\end{array}$ & Cyanobacteria \\
\hline A0A533NF66 & EBE85_21135 & Hormoscilla sp. GUM007 & Cyanobacteria \\
\hline A0A479ZZ55 & SR1949_29190 & Sphaerospermopsis reniformis & Cyanobacteria \\
\hline A0A357A498 & DD761_02610 & Cyanobacteria bacterium UBA11691 & Cyanobacteria \\
\hline A0A1Z4QDW0 & NIES4074_07940 & Cylindrospermum sp. NIES-4074 & Cyanobacteria \\
\hline K9R4C7 & Riv7116_0136 & Rivularia sp. PCC 7116 & Cyanobacteria \\
\hline A0A3S0ZZ73 & PCC6912_44900 & Chlorogloeopsis fritschii PCC 6912 & Cyanobacteria \\
\hline A0A3C0NJT8 & DCP31_40620 & Cyanobacteria bacterium UBA8543 & Cyanobacteria \\
\hline B2J6X7 & Npun_R5598 & Nostoc punctiforme PCC 73102 & Cyanobacteria \\
\hline A0A0C1NCV3 & DA73_0218765 & Tolypothrix bouteillei VB521301 & Cyanobacteria \\
\hline A0A1Z4S904 & NIES4103_38540 & Nostoc sp. NIES-4103 & Cyanobacteria \\
\hline A0A2K8SZ63 & C0091_06032 & Nostoc flagelliforme CCNUN1 & Cyanobacteria \\
\hline A0A3N6PGG7 & D5R40_05450 & Okeania hirsuta & Cyanobacteria \\
\hline A0A0C2QMV0 & SD80_01695 & Scytonema tolypothrichoides VB-61278 & Cyanobacteria \\
\hline Q3M6C5 & Ava_3856 & $\begin{array}{l}\text { Trichormus variabilis strain ATCC } \\
29413\end{array}$ & Cyanobacteria \\
\hline A0A1Z4ND62 & NIES3974_02980 & Calothrix sp. NIES-3974 & Cyanobacteria \\
\hline A0A0D8ZR72 & UH38_14315 & Aliterella atlantica CENA595 & Cyanobacteria \\
\hline A0A2T1LWM6 & C7H19_13915 & Aphanothece hegewaldii CCALA 016 & Cyanobacteria \\
\hline K9XU47 & Sta7437_1637 & Stanieria cyanosphaera PCC 7437 & Cyanobacteria \\
\hline A0A2Z6D2K3 & NIES2109_59170 & Nostoc sp. HK-01 & Cyanobacteria \\
\hline A0A5P8W9G9 & GXM_06696 & Nostoc sphaeroides CCNUC1 & Cyanobacteria \\
\hline
\end{tabular}




\begin{tabular}{|l|l|l|l|}
\hline A0A1S6LXZ0 & mylE & $\begin{array}{l}\text { Nostoc commune var. flagelliforme } \\
\text { QSY1 }\end{array}$ & Cyanobacteria \\
\hline A0A1Z4LFB5 & NIES25_64150 & Nostoc linckia NIES-25 & Cyanobacteria \\
\hline A0A4D9CF37 & BLD44_013555 & Mastigocladus laminosus UU774 & Cyanobacteria \\
\hline A0A1B2CWF7 & mysC & Heteroscytonema crispum UCFS10 & Cyanobacteria \\
\hline A0A0C1N3Z4 & DA73_0239150 & Tolypothrix bouteillei VB521301 & Cyanobacteria \\
\hline A0A2L2N6B5 & NPM_2790 & $\begin{array}{l}\text { Nostoc sp. 'Peltigera membranacea } \\
\text { cyanobiont' N6 }\end{array}$ & Cyanobacteria \\
\hline A0A1Z4Q915 & NIES4073_76020 & Scytonema sp. NIES-4073 & Cyanobacteria \\
\hline A0A2Z5VN68 & mysC & Nostoc commune KU002 & Cyanobacteria \\
\hline A0A1Z4UKN2 & NIES73_09950 & $\begin{array}{l}\text { Sphaerospermopsis kisseleviana NIES- } \\
\text { 73 }\end{array}$ & Cyanobacteria \\
\hline A0A5Q0GJK5 & EH233_17470 & Anabaena sp. YBS01 & Cyanobacteria \\
\hline A0ZIV3 & NSP_23010 & Nodularia spumigena CCY9414 & Cyanobacteria \\
\hline A0A3S1ANM2 & DSM106972_036000 & Calothrix desertica PCC 7102 & Cyanobacteria \\
\hline
\end{tabular}

Table S2. Bioinformatic analysis of the MAA gene cluster from Nostoc linkia NIES-25.

\begin{tabular}{|c|c|c|c|c|c|}
\hline Gene name & $\begin{array}{l}\text { Protein } \\
\text { accession }\end{array}$ & Size $^{1}$ & Homolog, origin & $|\mathrm{ID} / \mathrm{S}|^{2}$ & Predicted function \\
\hline NIES25_64110 & BAY79923.1 & 267 & $\begin{array}{l}\text { WP_190955827.1, } \\
\text { Nostoc }\end{array}$ & 98/99 & $\begin{array}{l}\text { Phytanoyl-CoA } \\
\text { dioxygenase }\end{array}$ \\
\hline NIES25_64120 & BAY79924.1 & 485 & $\begin{array}{l}\text { WP_190955828.1, } \\
\text { Nostoc }\end{array}$ & $94 / 97$ & $\begin{array}{l}\text { Major facilitator } \\
\text { transporter }\end{array}$ \\
\hline NIES25_64130 & BAY79925.1 & 410 & $\begin{array}{l}\text { RCJ25793.1, } \\
\text { Nostoc sp. ATCC } \\
43529\end{array}$ & $98 / 99$ & $\begin{array}{l}\text { Sedoheptulose 7- } \\
\text { phosphate cyclase }\end{array}$ \\
\hline NIES25_64140 & BAY79926.1 & 278 & $\begin{array}{l}\text { WP_190955830.1, } \\
\text { Nostoc }\end{array}$ & 95/97 & $\begin{array}{l}\text { Class I SAM-dependent } \\
\text { methyltransferase }\end{array}$ \\
\hline NIES25_64150 & BAY79927.1 & 464 & $\begin{array}{l}\text { WP_190955831.1, } \\
\text { Nostoc }\end{array}$ & $97 / 98$ & ATP-grasp ligase \\
\hline NIES25_64160 & BAY79928.1 & 368 & $\begin{array}{l}\text { WP_190955832.1, } \\
\text { Nostoc }\end{array}$ & $93 / 96$ & $\begin{array}{l}\text { D-alanine-D-alanine } \\
\text { ligase }\end{array}$ \\
\hline NIES25_64170 & BAY79929.1 & 257 & $\begin{array}{l}\text { RCJ25797.1, } \\
\text { Nostoc sp. ATCC } \\
43529\end{array}$ & $98 / 98$ & $\begin{array}{l}\text { short-chain } \\
\text { dehydrogenase/reductase }\end{array}$ \\
\hline
\end{tabular}

Note: ${ }^{1}$ amino acid; ${ }^{2}$ identities/similarities (\%). 
Table S3. Comparison of ${ }^{1} \mathrm{H}\left(600 \mathrm{MHz}, \mathrm{D}_{2} \mathrm{O}\right)$ and ${ }^{13} \mathrm{C}\{1 \mathrm{H}\}\left(151 \mathrm{MHz}, \mathrm{D}_{2} \mathrm{O}\right)$ chemical shifts of palythine-Thr determined in the current work and a recent report. ${ }^{1}$

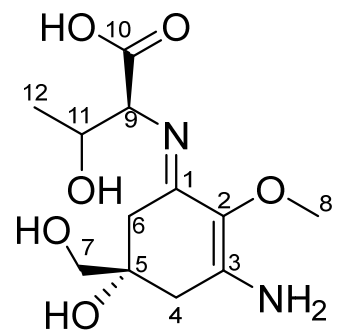

\begin{tabular}{|c|c|c|c|c|c|}
\hline \multirow[b]{2}{*}{ Position } & \multicolumn{2}{|r|}{ palythine-Thra } & \multicolumn{3}{|c|}{ literature $^{a}$} \\
\hline & $\delta_{\mathrm{c}}$, type & $\delta_{\mathrm{H}}(\mathrm{J}$ in $\mathrm{Hz})$ & $\delta_{\mathrm{C}}$, type & $\delta_{\mathrm{H}}(\mathrm{J}$ in $\mathrm{Hz})$ & \\
\hline $\begin{array}{l}1 \\
2\end{array}$ & $\begin{array}{l}163.8, \mathrm{C} \\
127.7, \mathrm{C}\end{array}$ & & $\begin{array}{l}163.8, \mathrm{C} \\
127.7, \mathrm{C}\end{array}$ & & \\
\hline 3 & 163.8, C & & 163.8, C & & \\
\hline 4 & $38.6, \mathrm{CH}_{2}$ & $2.97(17.1, \mathrm{~d}) 2.71(17.1,1.4, \mathrm{dd})$ & $38.6, \mathrm{CH}_{2}$ & $2.96(17.4, d)$ & $2.71(17.4, d)$ \\
\hline 5 & $74.2, \mathrm{C}$ & & $74.1, \mathrm{C}$ & & \\
\hline 6 & $36.6, \mathrm{CH}_{2}$ & $2.93(17.5, \mathrm{~d}) 2.77(17.5,1.3, \mathrm{dd})$ & $36.7, \mathrm{CH}_{2}$ & $2.92(17.4, d)$ & $2.77(17.4, d)$ \\
\hline 7 & $70.2, \mathrm{CH}_{2}$ & $3.58, \mathrm{~s}$ & $70.2, \mathrm{CH}_{2}$ & $3.58, \mathrm{~s}$ & \\
\hline 8 & $62.0, \mathrm{CH}_{3}$ & $3.69, \mathrm{~s}$ & $62.1, \mathrm{CH}_{3}$ & $3.69, \mathrm{~s}$ & \\
\hline 9 & $67.4, \mathrm{CH}$ & $4.08(4.6, d)$ & $67.4, \mathrm{CH}$ & $4.08(4.8, d)$ & \\
\hline 10 & $177.9, \mathrm{C}$ & & $177.9, \mathrm{C}$ & & \\
\hline 11 & $70.9, \mathrm{CH}$ & 4.32, $\mathrm{m}$ & $70.9, \mathrm{CH}$ & 4.32, $\mathrm{m}$ & \\
\hline 12 & $22.2, \mathrm{CH}_{3}$ & $1.26(6.5, d)$ & $22.2, \mathrm{CH}_{3}$ & $1.26,(6.6 \mathrm{~d})$ & \\
\hline
\end{tabular}

a: $\mathrm{D}_{2} \mathrm{O}$

Table S4. Primers used to construct refactored BGCs and express MysD.

\begin{tabular}{|c|c|}
\hline Primer & Sequence (5'-3') \\
\hline MysA-Ncol-F & CATGCCATGGTGAGCATTGTTCAAACAA \\
\hline MysB-Pstl-R & CATGCTGCAGTCACGCAGTTCTGCGGATA \\
\hline MysC-Kpnl-F & CGTCGGTACCATGGCACAATCTATTTCCG \\
\hline MysC-Xhol-R & CAGACTCGAGCTAATCCCCACCCAATTCCA \\
\hline MysD-Ndel-F & CATGCATATGCCAGTACTTCGTATC \\
\hline MysD-Xhol-R & CATGCTCGAGCTAAATCATTTGTGAAAGCT \\
\hline MysH-Ncol-F & TAATAAGGAGATATACCATGGTGAAGGTAGACACACA \\
\hline MysH-Pstl- R & GCAAGCTTGTCGACCTGCAGTCGATGTACTTGAACTCTAG \\
\hline SDR-Ndel-F & TAAGAAGGAGATATACATATGGCTTCTCTAGAAAATCA \\
\hline SDR-Xhol-R & GTTTCTTTACCAGACTCGAGCTAAGTGCGCCGATTAACTA \\
\hline
\end{tabular}




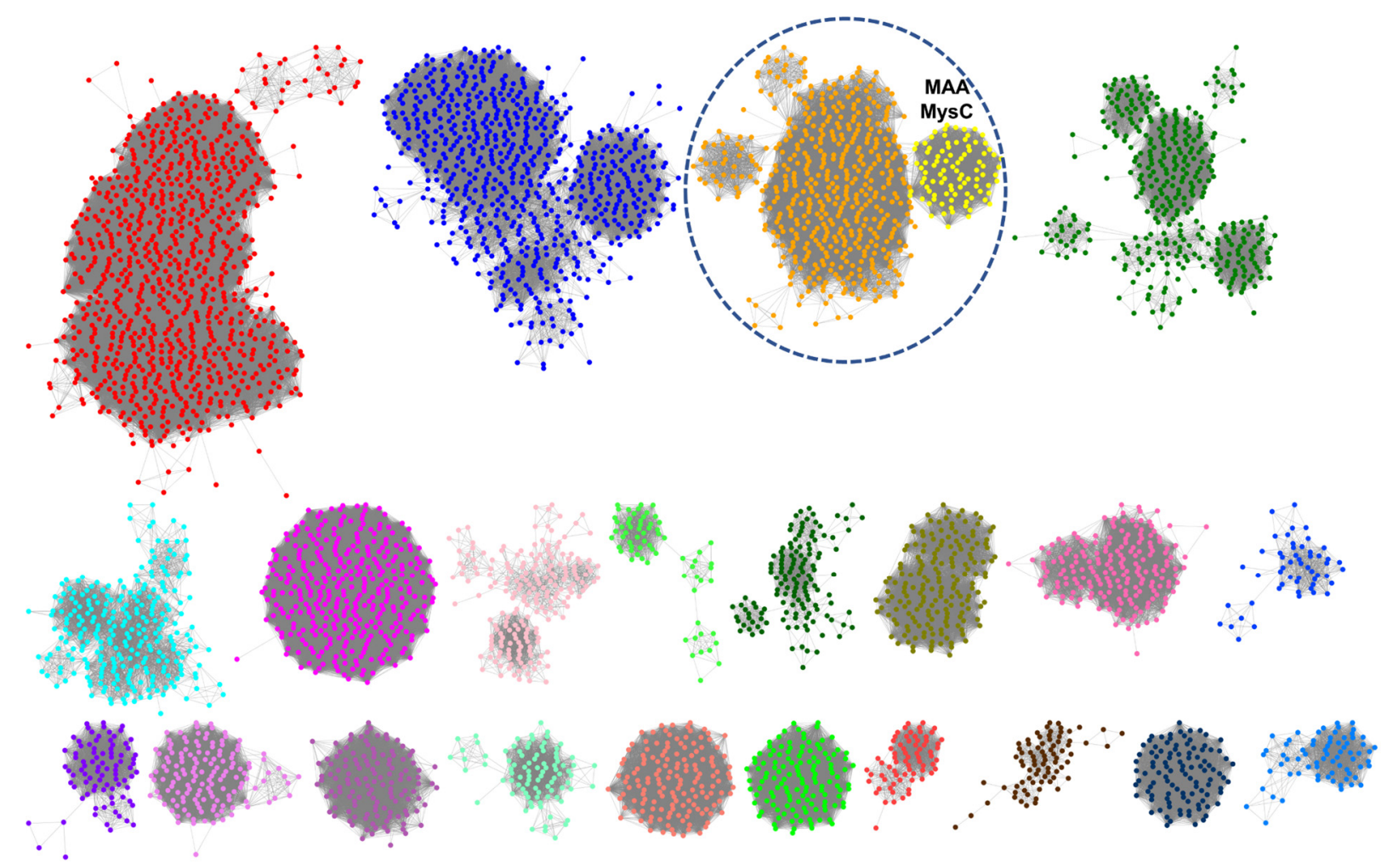

Figure S1. Sequence similarity network (SSN) analysis ${ }^{2}$ of protein family \#02655 in the Pfam database identified 22 distinct clusters with a sequence identity of $>35 \%$ (filter value 56 ) of MysC proteins. The cluster with 92 MysC homologs as a subcluster is circled. 
A0A3 67QPY5

A0A2Z6D3B5

A0A2T1LWM2

A0A2L2NS5 2

A0A2 C6TQQ 8

A0A2 52E419

A0A1Z4LFC6

A0A1Z4 IIA4

A0A1Z4HWL1

A0A1U7I924

A0A367QPY5

A0A2 Z6D3B5

A0A2 T1LWM2

A0A2L2NS52

A0A2 C6TQQ 8

A0A252E419

A0A1Z4LFC6

A0A1Z4 I IA4

A0A1Z4HWL1

A0A1U7I 924

A0A367QPY5 18

A0A2 Z6D3B5

A0A2T1LWM2

A0A2L2NS52

A0A2 C6TQQ8

A0A252E419

A0A1Z4LFC6

A0A1Z4IIA4

AOA1Z4HWL

A0A1U7I 924
61 GSVEHASSLWLVDADFRALAFESPLPTLAAQVLKSEKLNFLADGFFVKKPKTNGHIGWHN

61 GNVEHTNSLWLVDADFRALVFESPLATLAAQ LKSTKLNFLADGFFVKOPKATSRVGWHN

59 GDVVHDYGLWLKDNDFRDLVFKSPLAR AAQ MESET NFLCDGFFVKKAKADSHVGWHN

61 GHVEHTSSLWLTDADFRALAFESPLATLTAQVLKSKKLNFL DDFFVKKPKCETGVGWHN

61 ASAEHTSSLWLVDADFRALAFESPLPKLAVGVLKSEKLNFLADGFFVKR PEANGR GWHN

61 ASVEHTSSLWLVDPDFRALVFESPLSTAAQ L SEKLNFLADGFFVKKPKATSRVGWHN

61 GSAEHASS WLIDADFRALAFESPLPTLAAQVLKSKKLNFLADGFFVKKPESNGR I GWHN

61 GNVEHSNSLWLVDTDFRALVFESPLANLAAQFLKSTKLNFLADGFFVKNPKASSRVGWHN

61 ASVEHTSSLWLVDPDFRALVFESPLST AAQ LQSEKLNFLADGFFVKKPKATSRVGWHN

61 ASAEHTSNLWLVDADFRALAFESPLPTLAVQVLKSKKLNFLADGFFVKKPKSNSR GWHN 1

DLPYWE QGWQCCKIWLPLD VKQENGRLEYIKGSHQWGKELRERSNPSWFVEPEPHEII DLPYWPIQGWQCCKIWLALDKVNQQNGRLEYIKGSHRWGKELREDSNPAWFSQPESHE I DLPYWP VKGWKCCKIWLALDPVNQENGRLEYIKGSHLWNKWLRENSNVSWFSEPSYSDIL

21 DKSYWPIQGWQCCKIWLALD VNQENG LEYIK SHLWGKELREASDPSWFVEPEPHEI I

121 DLPYWP QGWQCCKIWLALD VKQENGRLEYIKGSHQWG ELRERSNPSWFVEPEPHEIL DLPYWPIQGWQFCKIWLALDNVNEENGRLEYIKGSHQWGKELREDSNPSWFVEPEPHE I DLPYWP QGWQCCKIWLALD VKQENGRLEYIKGSHQWGKELRERSNPSWF IEPEPHEIL DLPYWPIQGWQCCKIWLALDKVNQQNGRLEYIKGSHRWGKELREDSNPSWFSEPEPHE L DLPYWPIQGWQCCKIWLALDHVNEKNGRLEY IKGSHKWGKELREDSNPLWFVEPEPHE I DLPYWP IQGWQCCKIWLALD VNQENGRLEY IKGSHRWGKELRERSNPSWFVEPKPHEIL<smiles>C1C[Te]C1</smiles>

SWDMEAGDCLIHHFLTIHHSVTNISSTQRRA VTNWTGDDVTYYQRPKAWPF PLEEIDI SWDMEPGDCLVHHLLTIHHSVTNISSTQRRAVVTNWTGDDVTYYPRPKAWPFRPLDEID I YWDMEPGDALVHHFQTIHHS IGNTTYKSRRA VTNWTGDDVVYDPSPQTWPFQPIEEIGI SWDMEPGDCLVHHF TIHHSVRNTSSTRRRAVVINWTGDDVTYERRPNAWPFRPLEEIDI SWDMEAGDCLIHHFLTIHHSVTNKSSTQRRA VTNWTGDDVTYYQRPKAWPF KLEEIDL SWDMEPGDCLVHHLLTIHHSVTNISSRQRRAVVTNWTGDDVTYYPRLKAWPFRPLEEIDL SWDMEAGDCLIHHFLTIHHSVTNISSTQRRA VTNWTGDDVTYYQRPKAWPF PLEEIDI SWDMEPGDCLVHHLLTIHHSVTNISSTKRRAVVTNWTGDDVTHYPRPKAWPFRPLDEIDI SWNMEPGDCLVHHLLTIHHSVTNISSTQRRAVVTNWTGDDVTYYPRPKAWPFRS VEEIDL SWDMEAGDCLIHHFLTIHHSVTNISSRQRRAVVTNWTGDDVTYYQRPKAWPFKS IEEIDL

Figure S2. Sequence alignment of all phytanoyl-CoA dioxygenases identified in the GNN analysis revealed the conserved 2-His-1-carboxylate facial triad (His119, D121 and His198 for A0A367QPY5). 


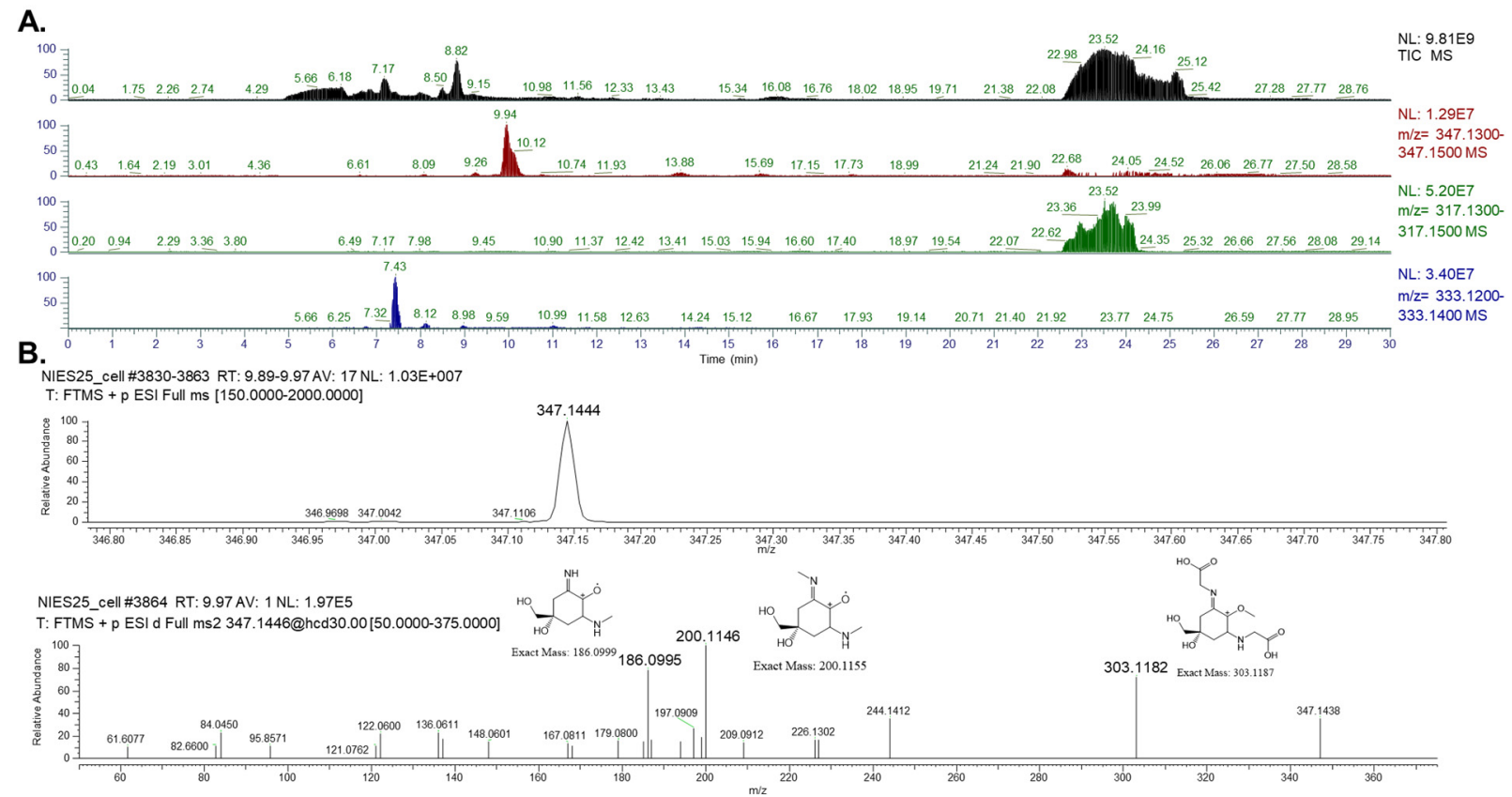

Figure S3. (A) TIC and EIC traces of methanolic extracts of $N$. linkia NIES-25 cells. Value ranges used to generate EIC traces represent the $\mathrm{m} / \mathrm{z}$ values of parental ions of porphyra-334 (calculated $[\mathrm{M}+\mathrm{H}]^{+}:$347.1449), shinorine (calculated $[\mathrm{M}+\mathrm{H}]^{+}:$333.1292), and MG-Ala (calculated $[\mathrm{M}+\mathrm{H}]^{+}$: 317.1343). Potential peaks for porphyra-334 and shinorine were observed. (B) HRMS and MS/MS spectra of putative porphyra-334 peak. Structures of fragment ions with $\mathrm{m} / \mathrm{z}$ values of 186.0995 , 200.1155 and 303.1182 were proposed. 
A.

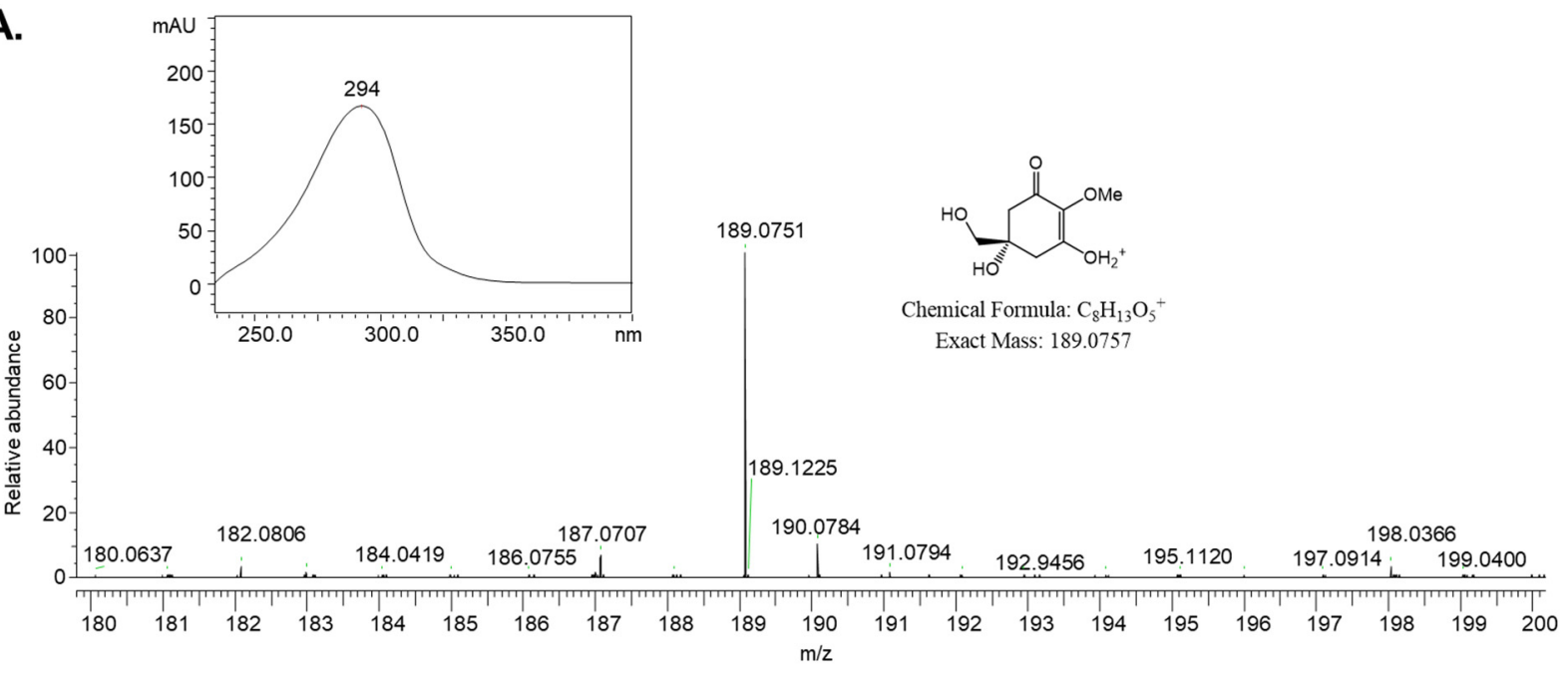

B.

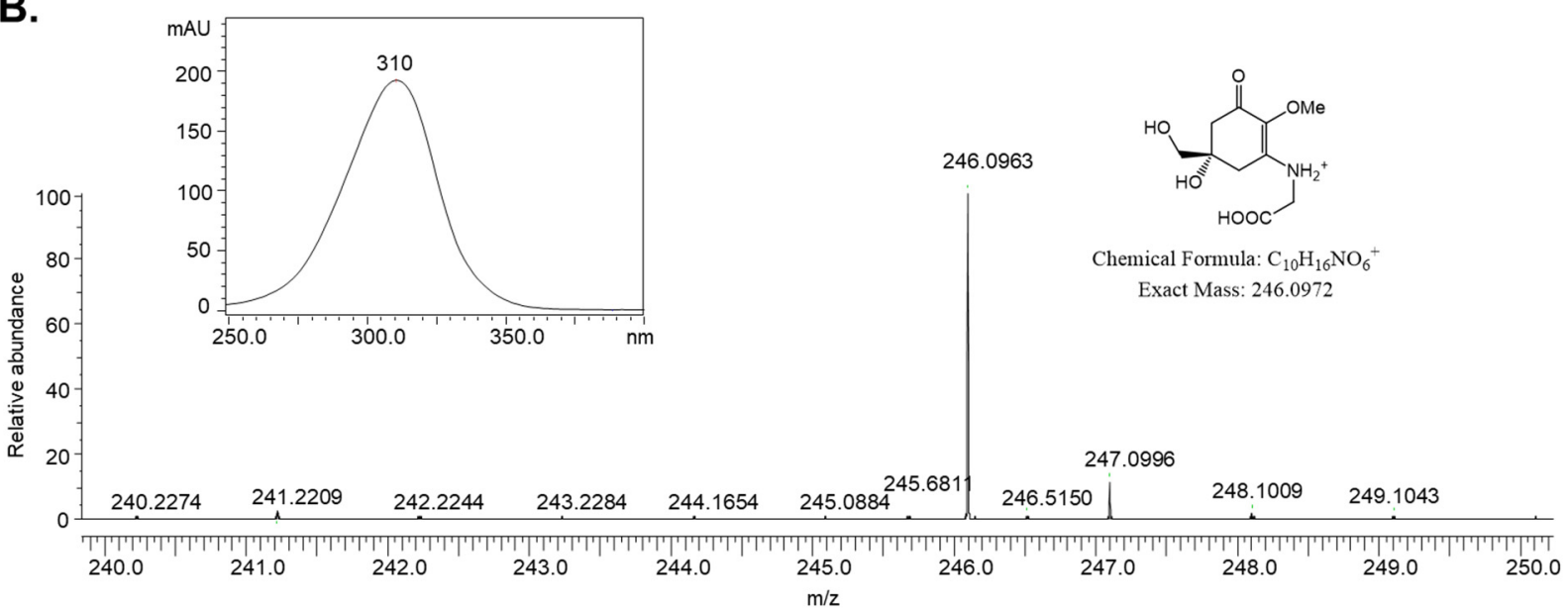

Figure S4. The maximal UV absorbance and HRMS spectra of 4-DG (A) and MG (B) produced in engineered $E$. coli. 

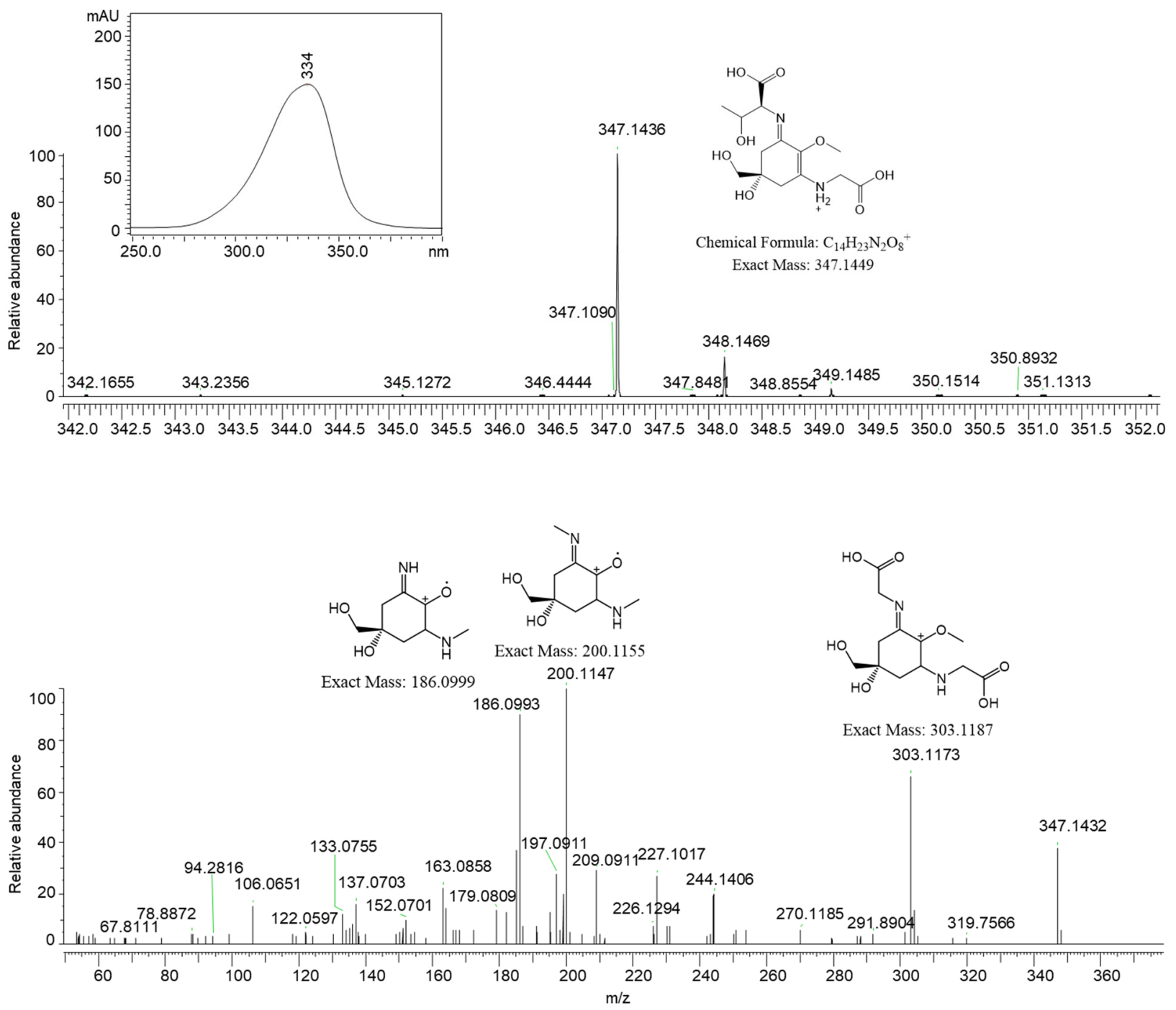

Figure S5. The maximal UV absorbance, HRMS and MS/MS spectra of porphyra-334 produced in engineered $E$. coli. 

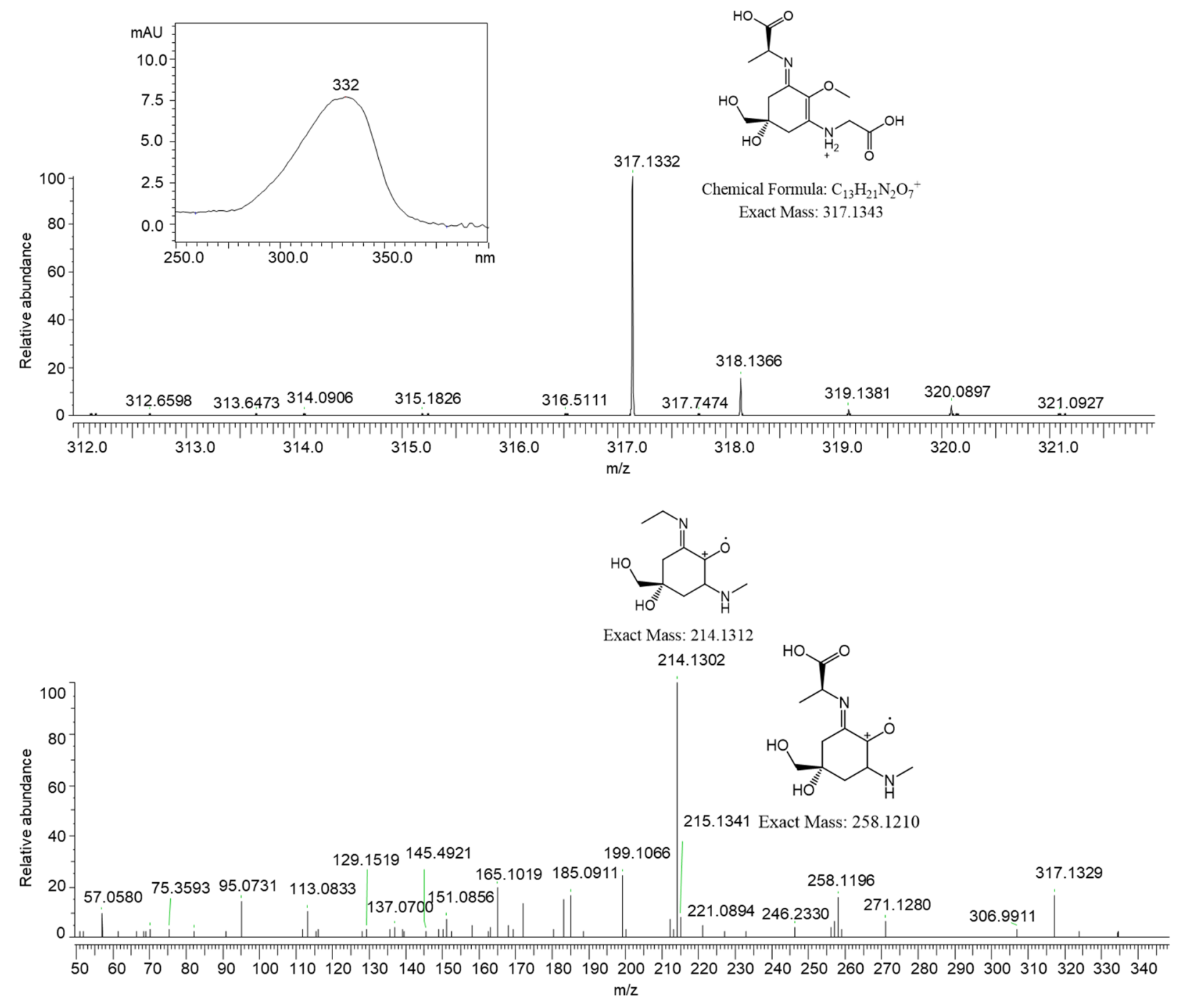

Figure S6. The maximal UV absorbance, HRMS and MS/MS spectra of MG-Ala produced in engineered $E$. coli. 

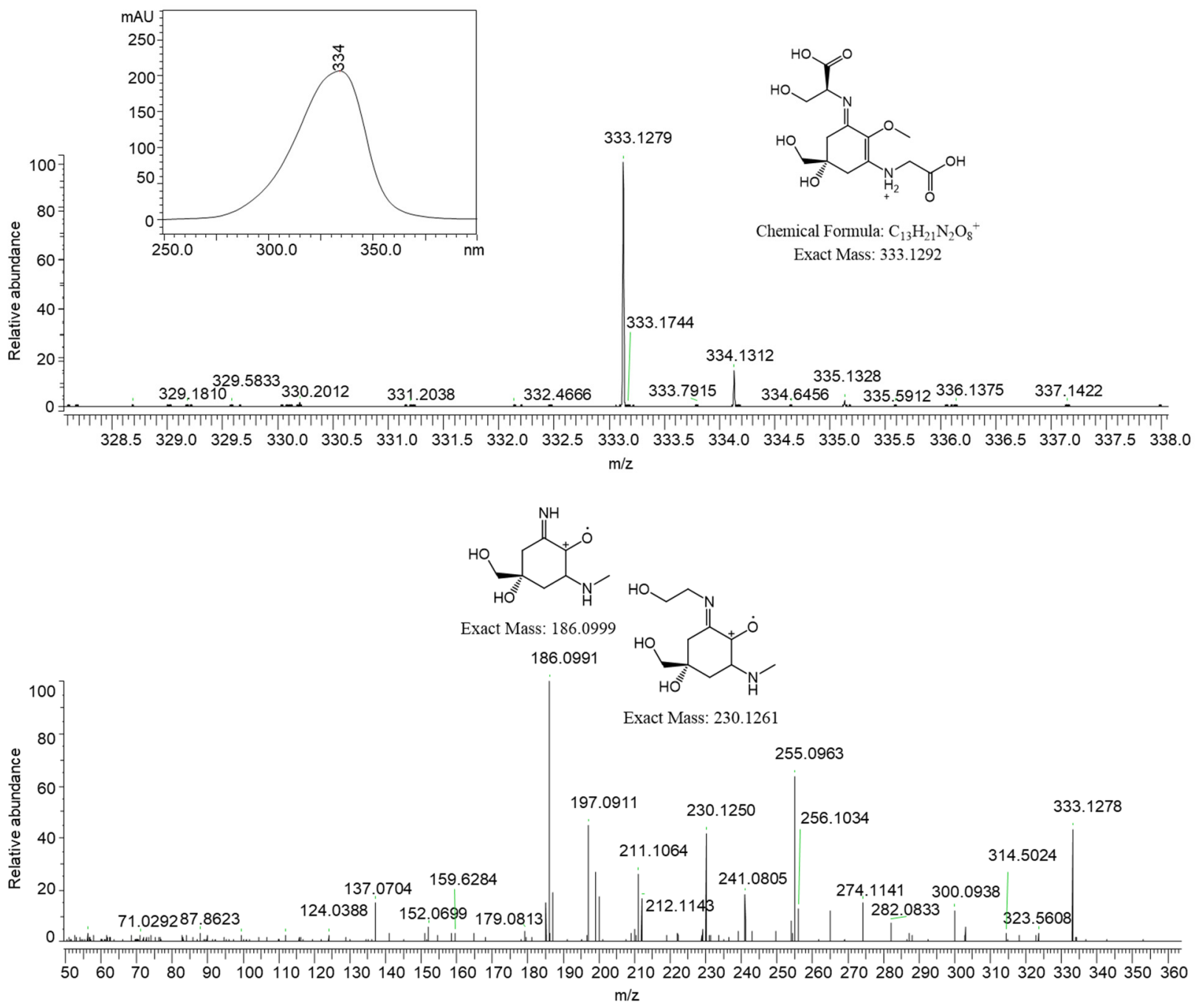

Figure S7. The maximal UV absorbance, HRMS and MS/MS spectra of shinorine produced in engineered E. coli. 


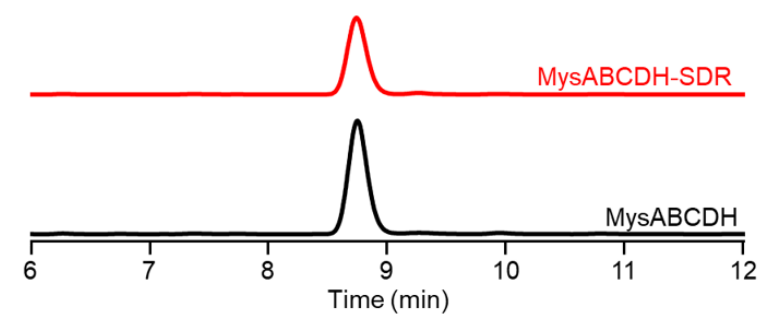

Figure S8. HPLC trace of methanolic extract of $E$. coli expressing mys $A B C D H$ (bottom) and mys $A B C D H$-sdr (top).
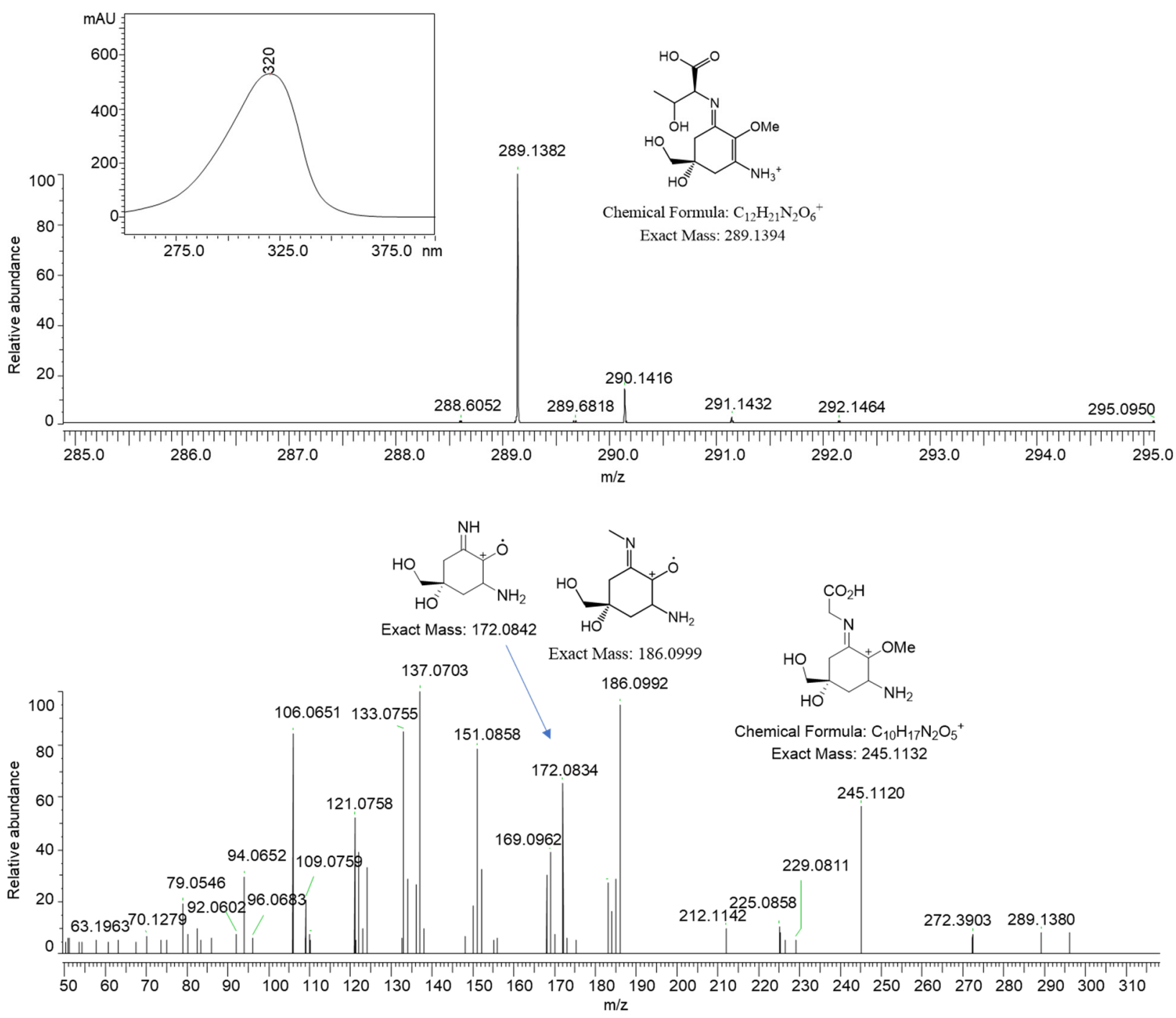

Figure S9. The maximal UV absorbance, HRMS and MS/MS spectra of palythine-Thr produced in engineered $E$. coli. 


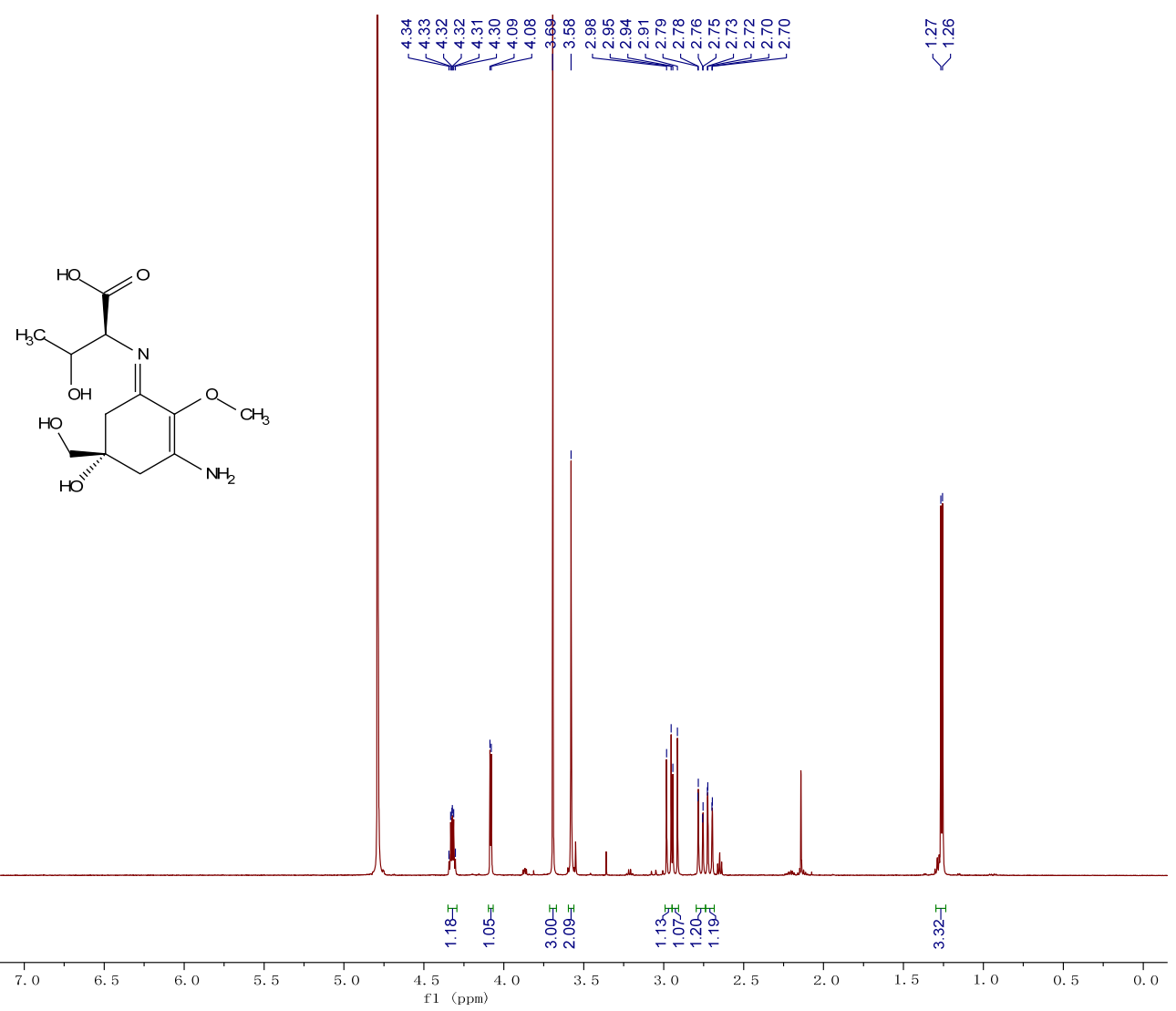

Figure S10. ${ }^{1} \mathrm{H}$ NMR spectrum of isolated palythine-Thr $\left(\mathrm{D}_{2} \mathrm{O}, 600 \mathrm{MHz}\right)$. Of note, a chemical shift of formic acid was observed. 


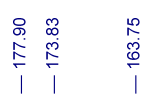

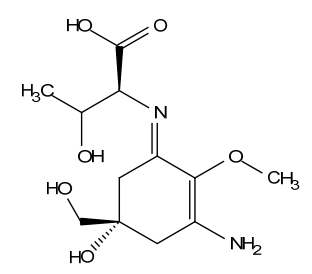

Formic acid
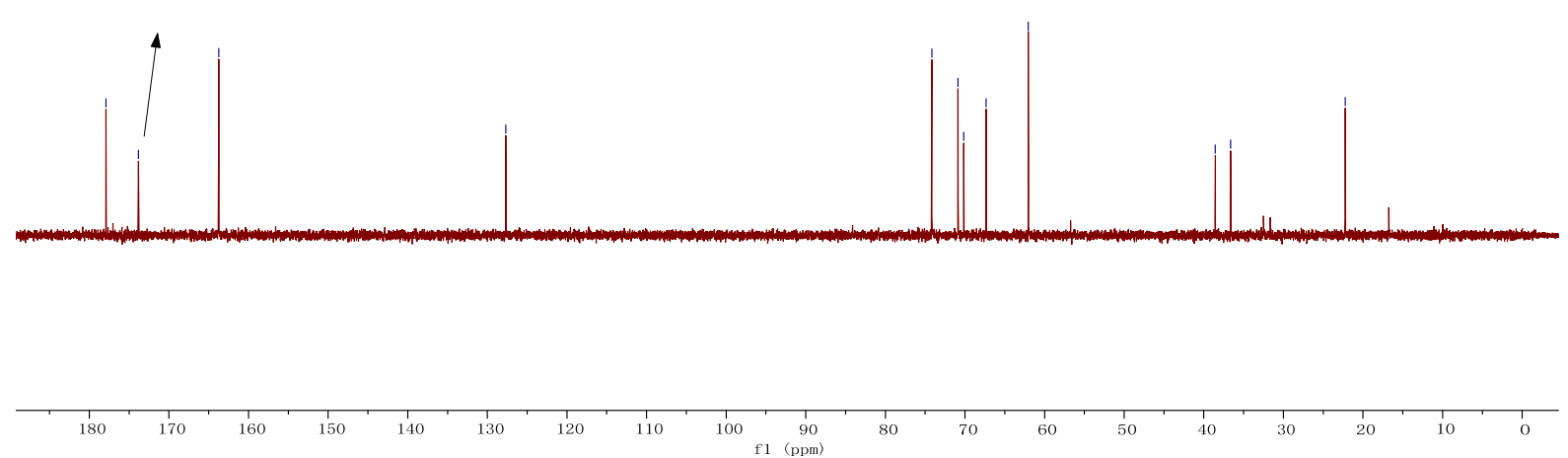

Figure $\mathrm{S} 11 .{ }^{13} \mathrm{C}\{1 \mathrm{H}\}$ NMR spectrum of isolated palythine-Thr $\left(\mathrm{D}_{2} \mathrm{O}, 151 \mathrm{MHz}\right)$. Of note, a chemical shift of formic acid was observed. 
A.

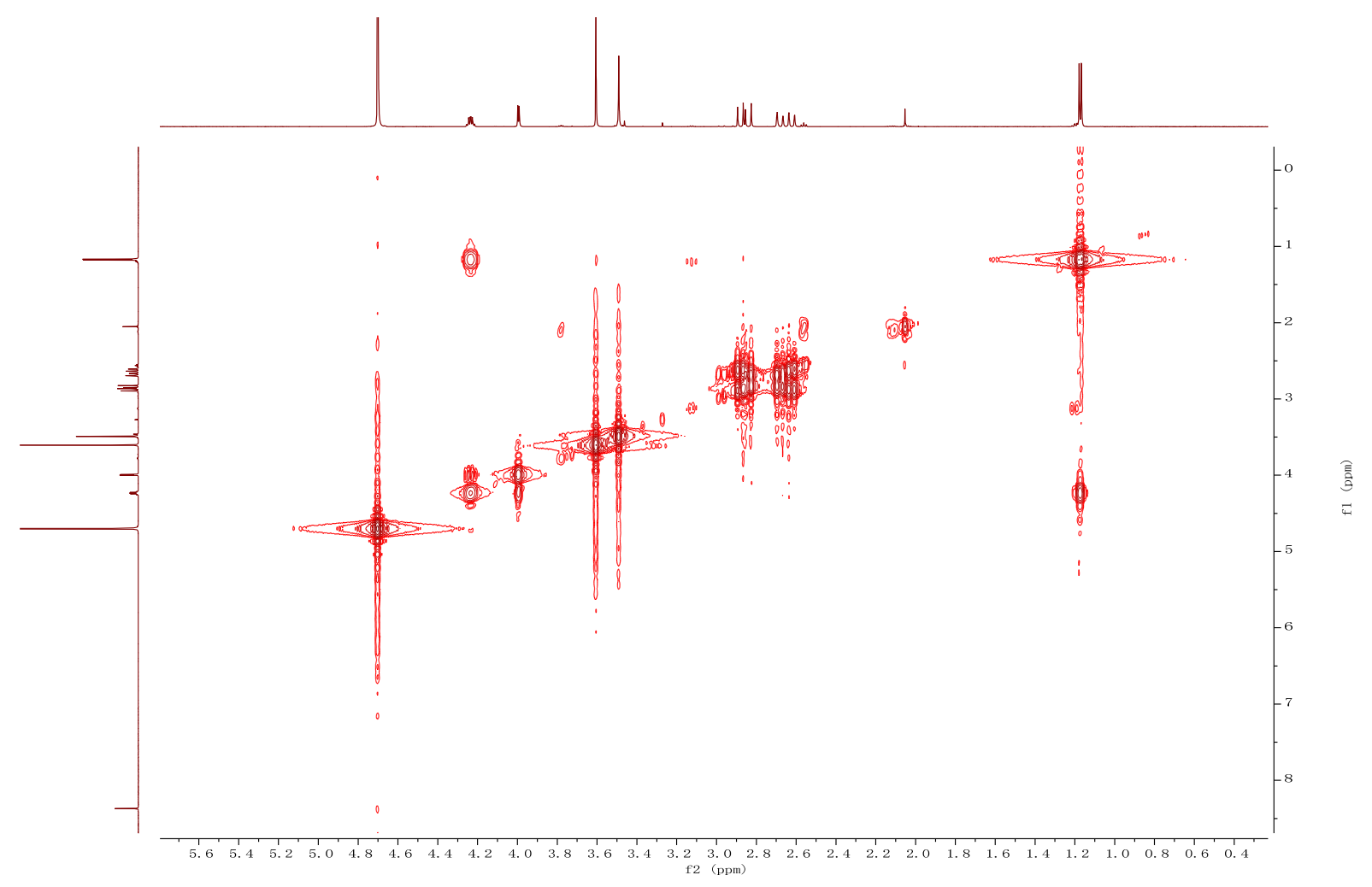

B.

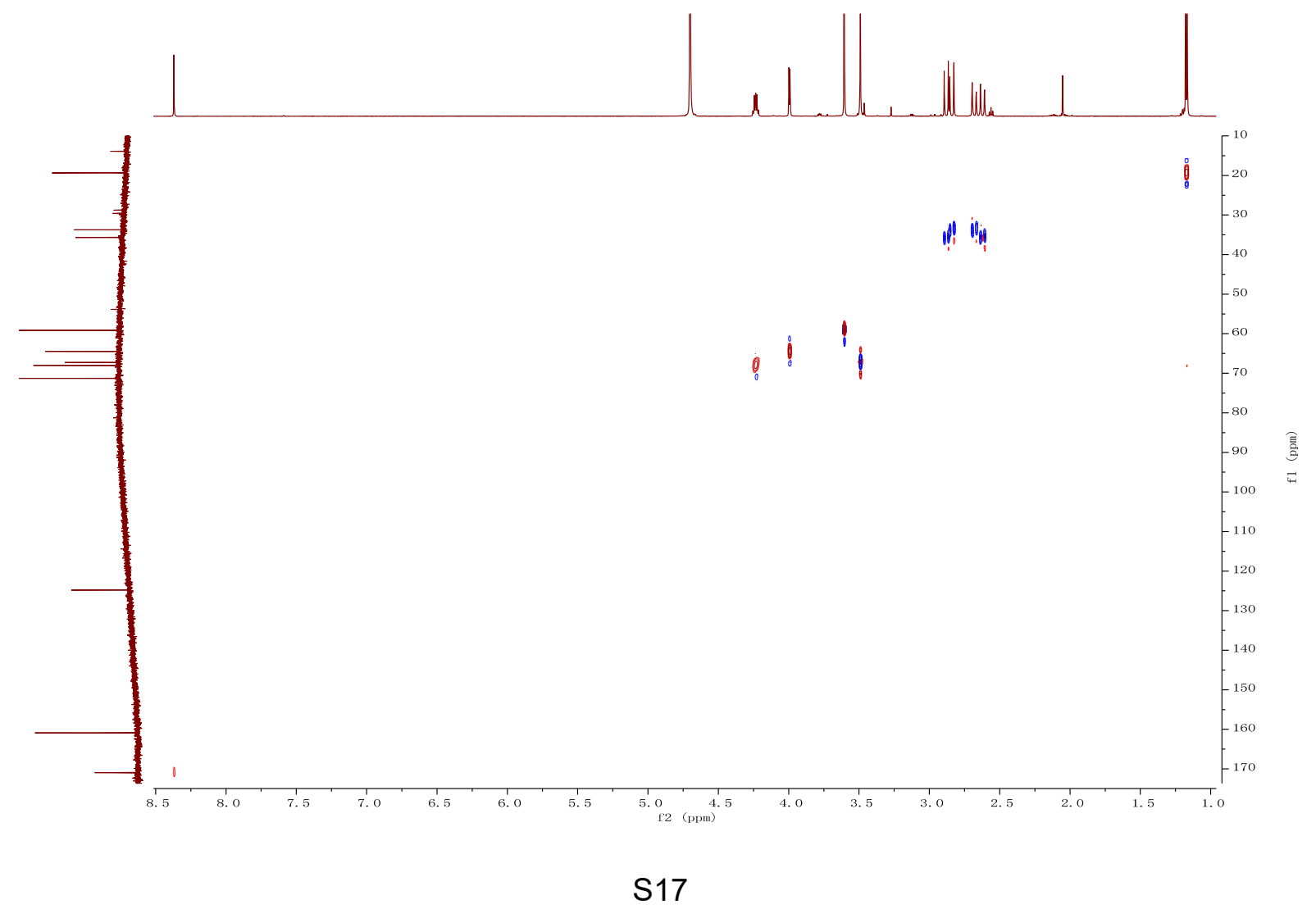


c.

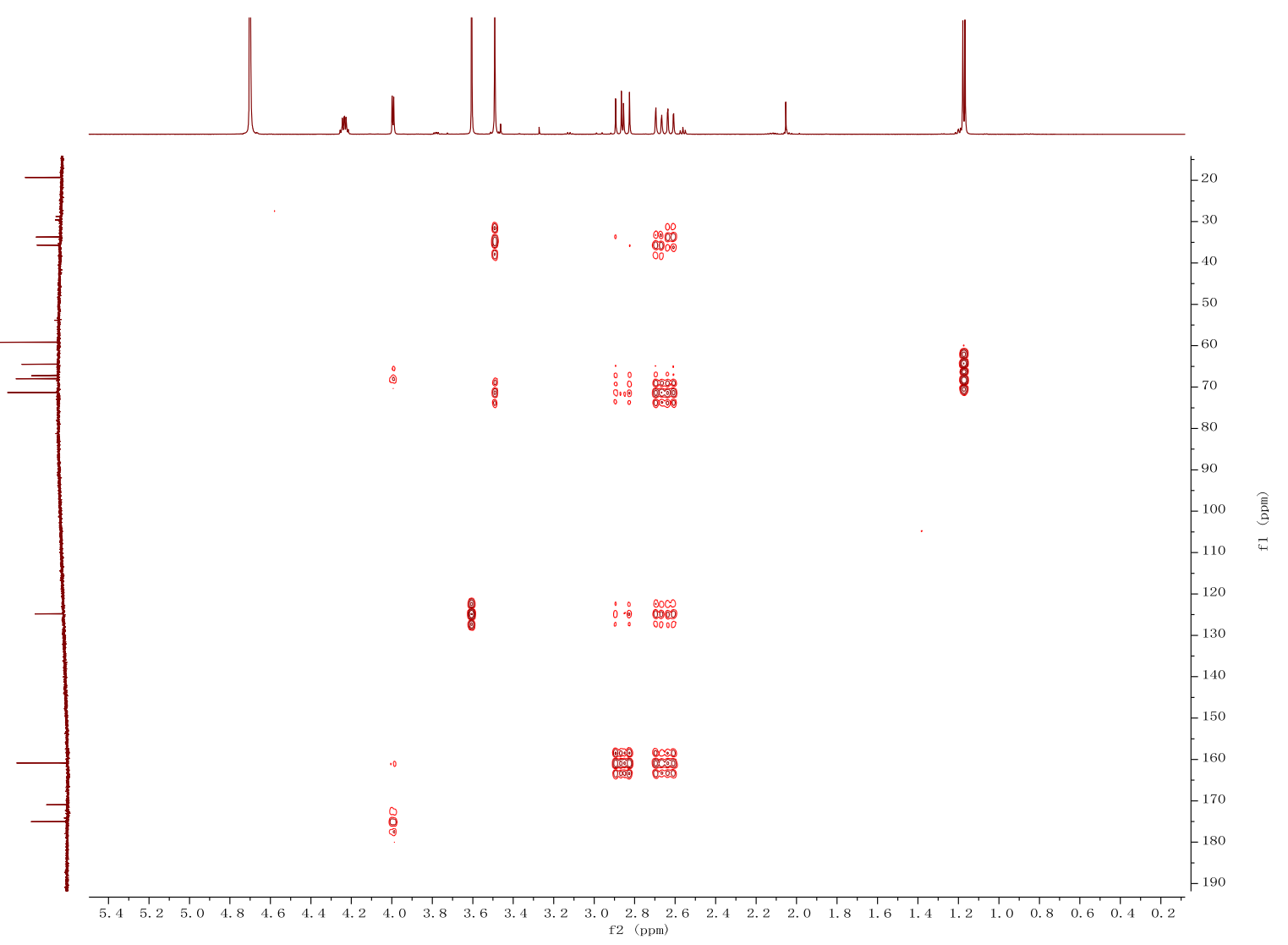

Figure S12. 2D NMR spectra of isolated palythine-threonine. A. ${ }^{1} \mathrm{H}-{ }^{-1} \mathrm{H}$ COSY; B. HSQC; C.HMBC. 
A.

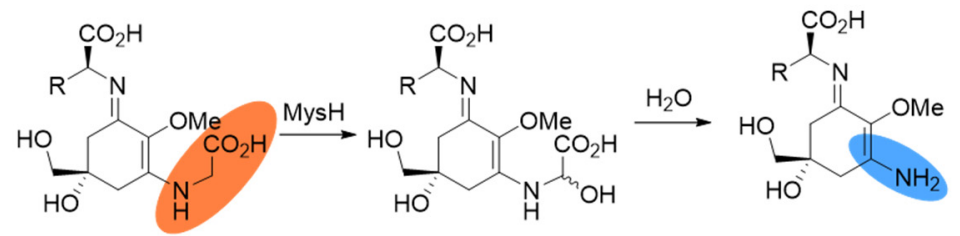

B.

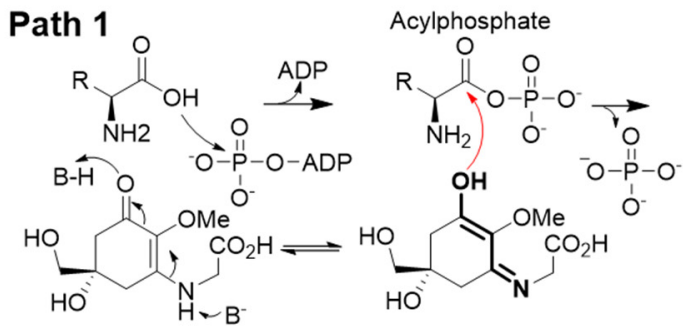

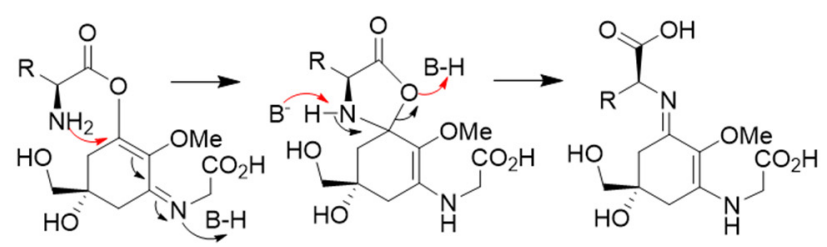

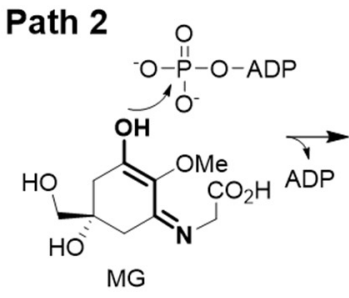

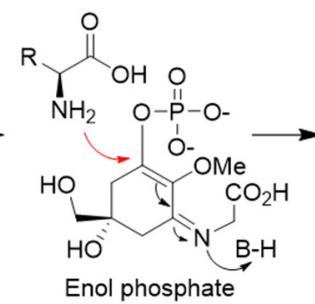

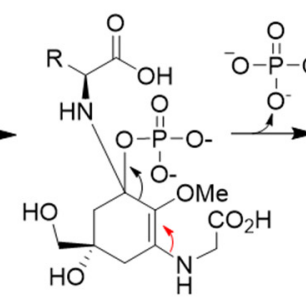

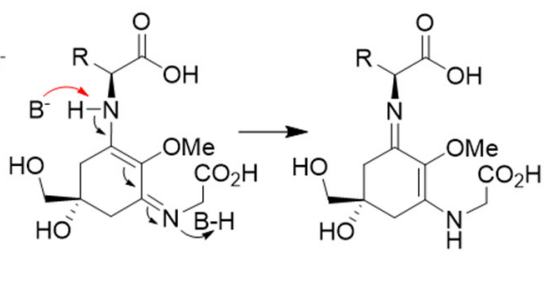

Figure S13. (A) A proposed pathway for conversion of disubstituted MAAs into palythines by MysH. (B) Proposed mechanisms of MysD-catalyzed amino acid ligation with MG. Its reaction can go through an acylphosphate (path 1) or enol phosphate intermediate (path 2). 

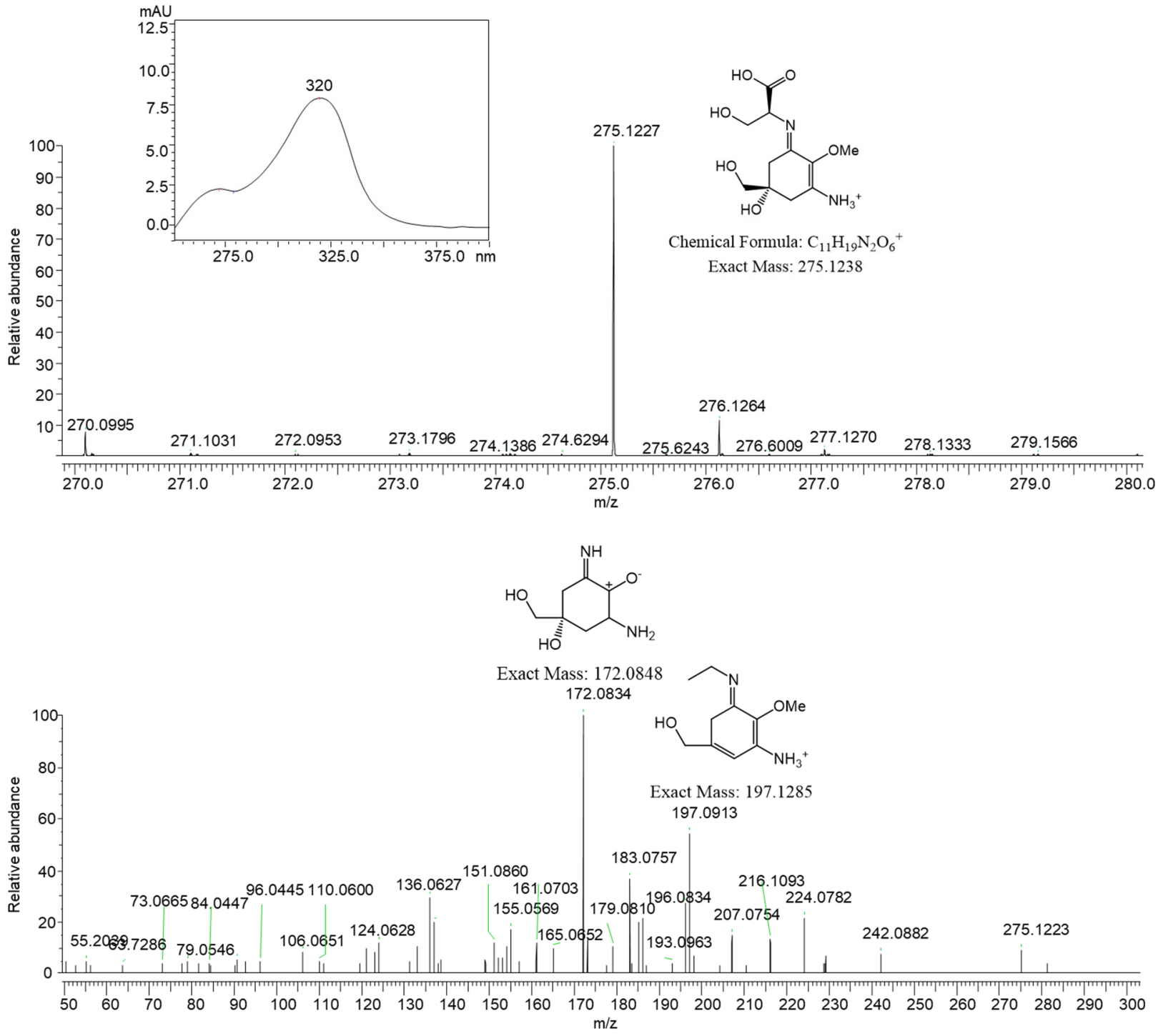

Figure S14. The maximal UV absorbance, HRMS and MS/MS spectra of palythine-Ser produced in engineered $E$. coli. 

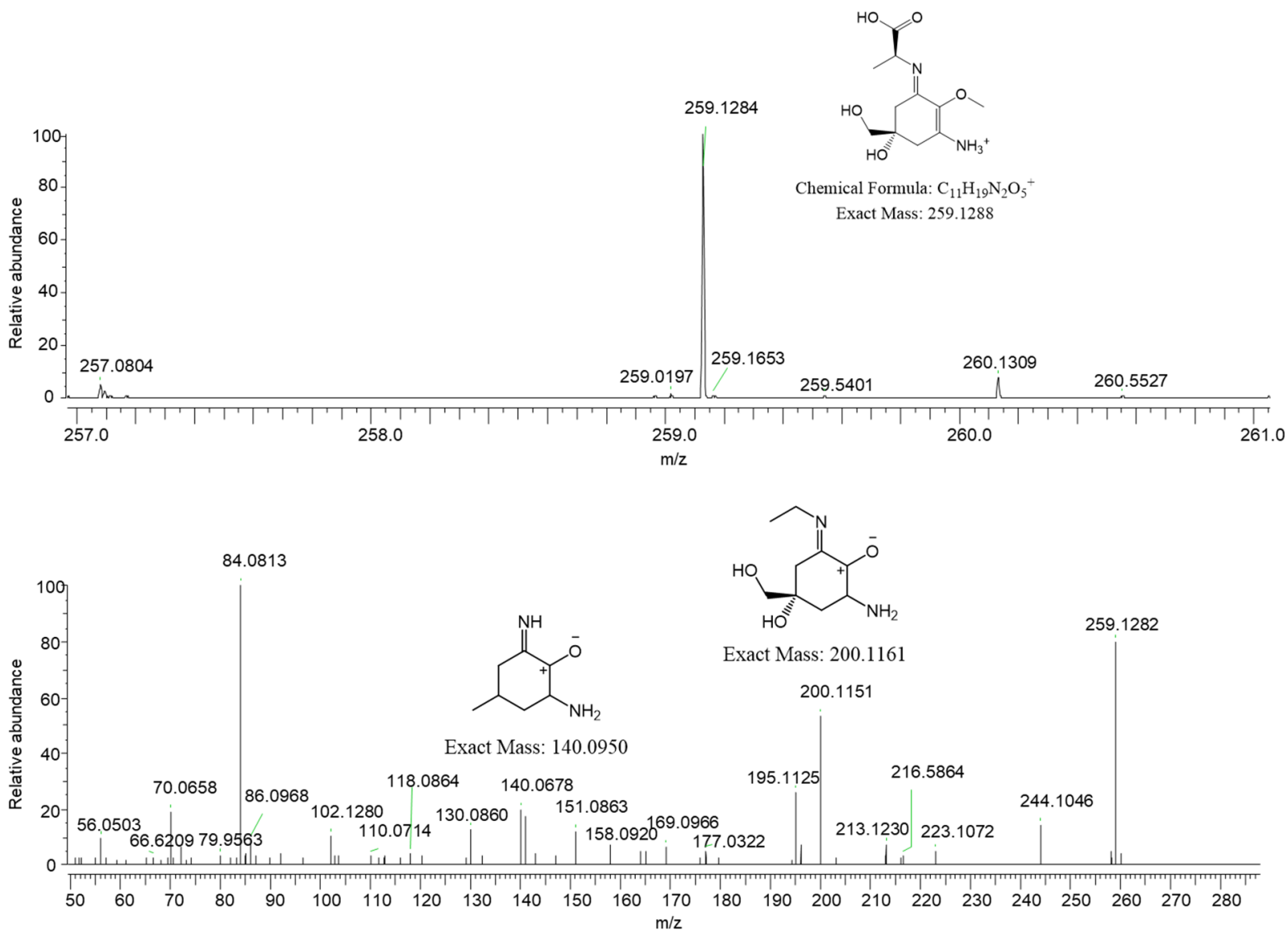

Figure S15. The HRMS and MS/MS spectra of palythine-Ala produced in engineered E. coli.

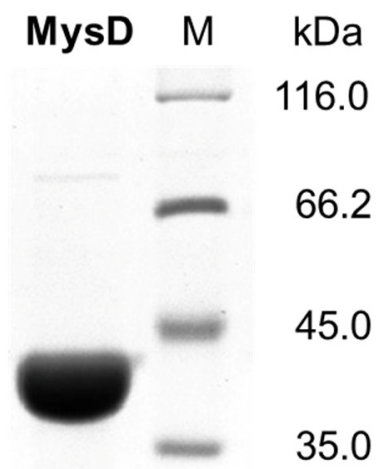

25.0

Figure S16. SDS-PAGE analysis of recombinant MysD. MysD showed the expected molecular weight at $42.9 \mathrm{kD}$. 

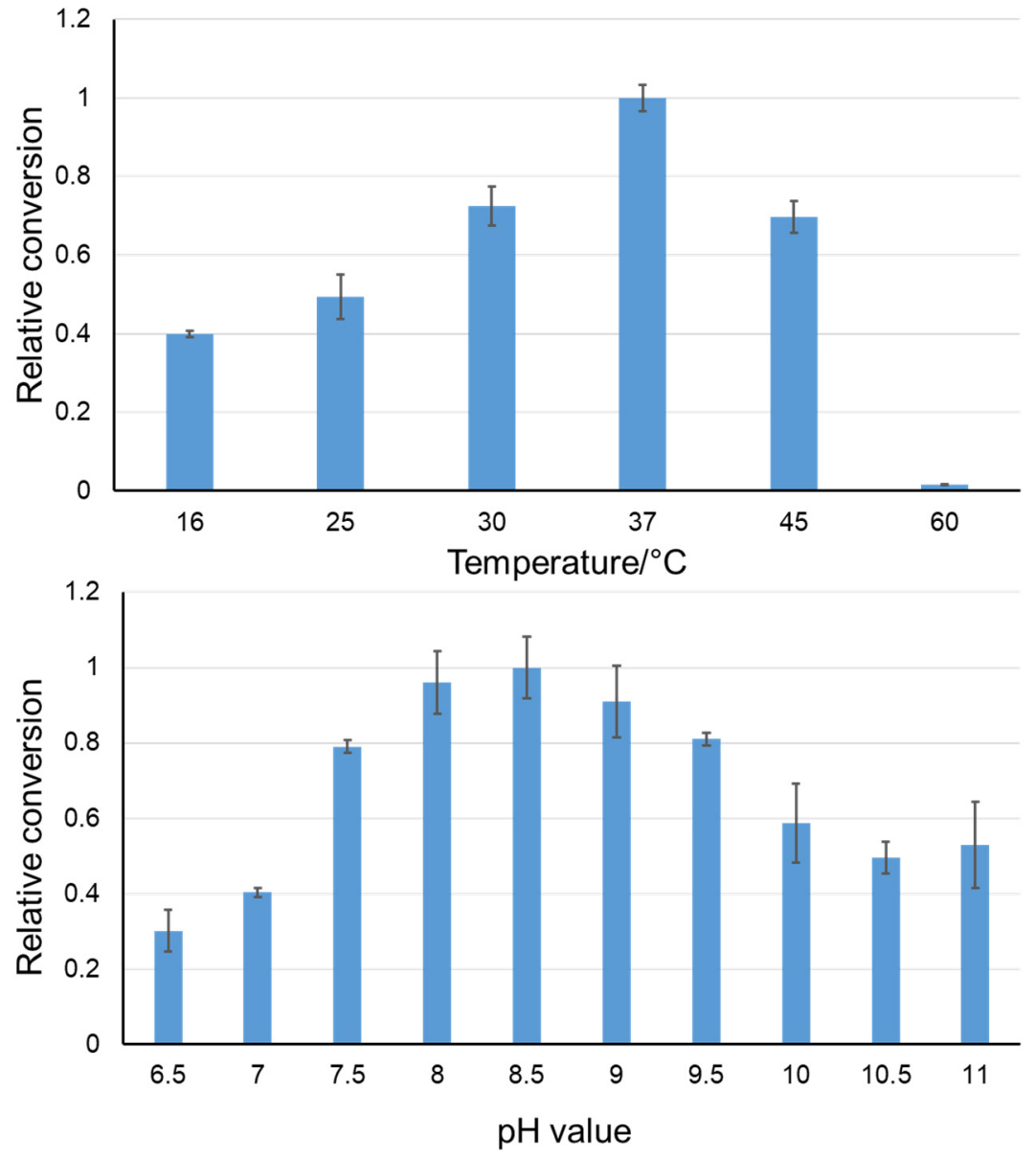

Figure S17. Determination of optimal temperature and $\mathrm{pH}$ for the MysD reaction. The reaction mixture contained $100 \mathrm{mM}$ buffer (pH 6.5 to 11), $10 \mathrm{mM} \mathrm{MgCl}_{2}, 5 \mathrm{mM} \mathrm{ATP}, 500 \mathrm{nM}$ MysD, $50 \mu \mathrm{M}$ $\mathrm{MG}$, and $5 \mathrm{mM}$ Thr. The reaction was conducted incubated at 16 to $60{ }^{\circ} \mathrm{C}$ for $6 \mathrm{~min}$ and then quenched by incubation at $95^{\circ} \mathrm{C}$ for $10 \mathrm{~min}$. The highest conversion ratio of MG was set as $100 \%$ for normalizing other reactions. Data represent means $\pm \mathrm{s}$. d. of at least two independent experiments. 

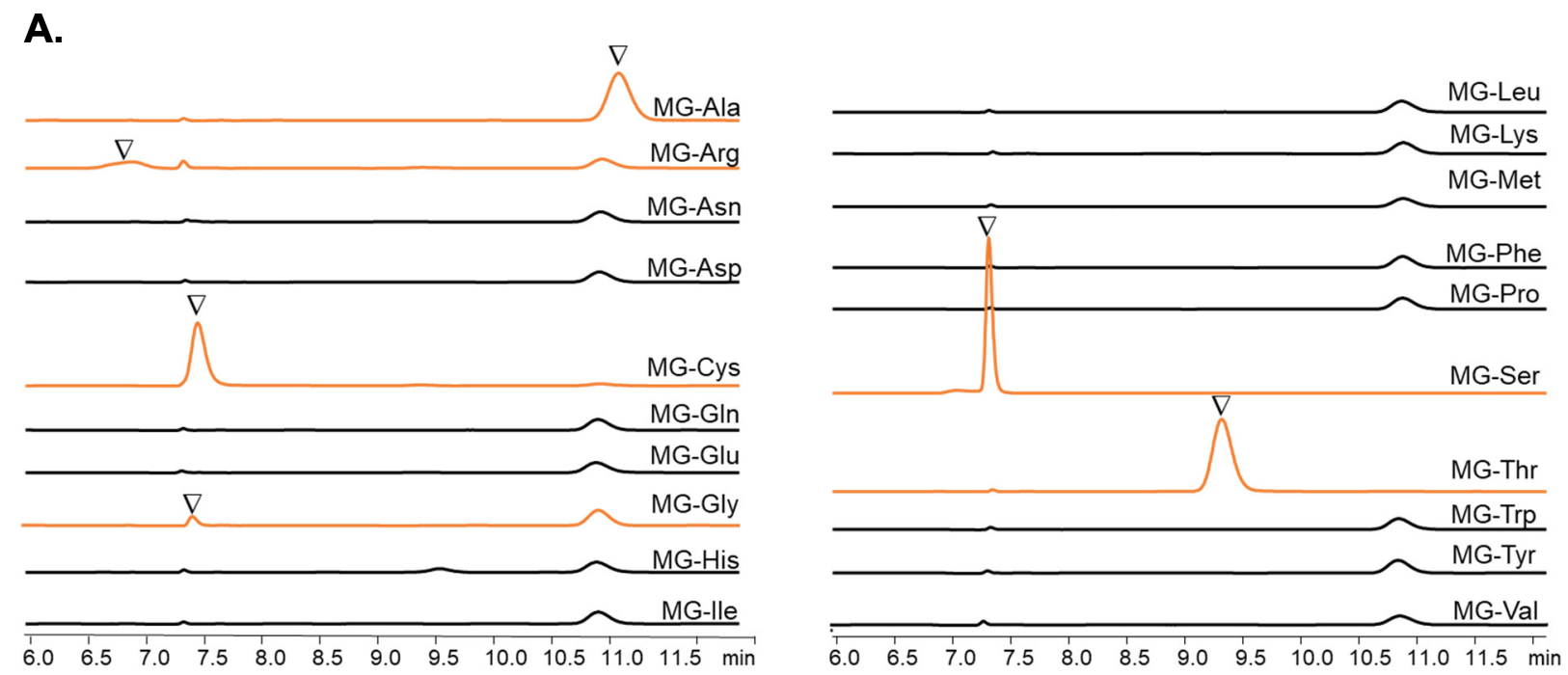

B.

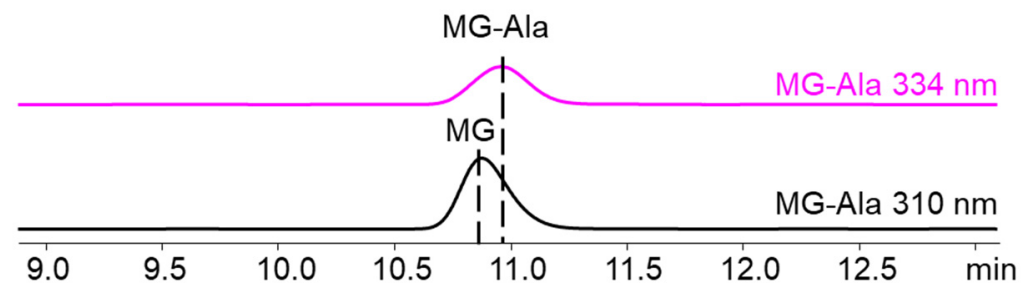

C.<smiles>COC1=C(NCC(=O)O)CC(CO)(CO)CC1=NC(C)C(=O)O</smiles>

MG-Ala<smiles>COC1=C(NCC(=O)O)C[C@](O)(CO)C/C1=N/[C@@H](CCCNC(=N)N)C(=O)O</smiles>

MG-Arg<smiles>COC1=C(NCC(=O)O)C[C@](O)(CO)CC1=N[C@@H](CS)C(=O)O</smiles>

MG-Cys<smiles>COC1=C(NCC(=O)O)C[C@](O)(CO)CC1=NCC(=O)O</smiles>

Mycosporine-2-Gly

Figure S18. (A) HPLC trace of the MysD reactions with MG and all 20 amino acids as substrates. The mixtures were separated on a Phenomenex Luna C8 5um column with mobile phases $0.1 \mathrm{M}$ TEAA (pH 7.0) and $2 \%$ methanol. The detection wavelength was $334 \mathrm{~nm}$. All disubstituted MAAs were labeled with $\nabla$ and their traces were in orange. (B) LC traces of the MysD reaction with LAla as substrate with the detection wavelengths of $334 \mathrm{~nm}$ and $310 \mathrm{~nm}$ (specific to MG). (C) Chemical structures of four disubstituted MAAs, Mg-Ala, MG-Arg, MG-Cys, and mycosporine-2Gly, produced in the MysD reaction. 

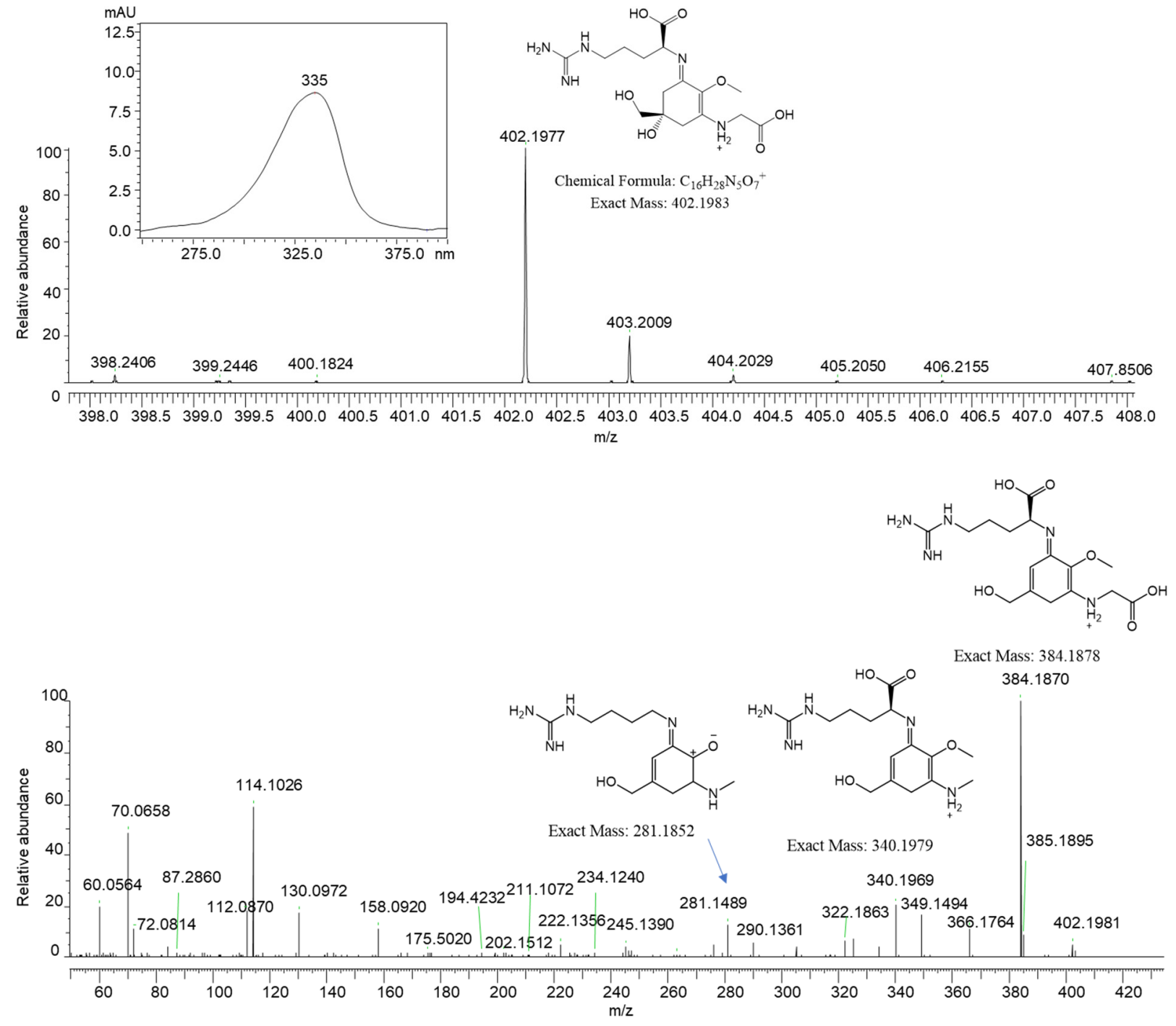

Figure S19. The maximal UV absorbance, HRMS and MS/MS spectra of MG-Arg produced in the MysD reaction. 


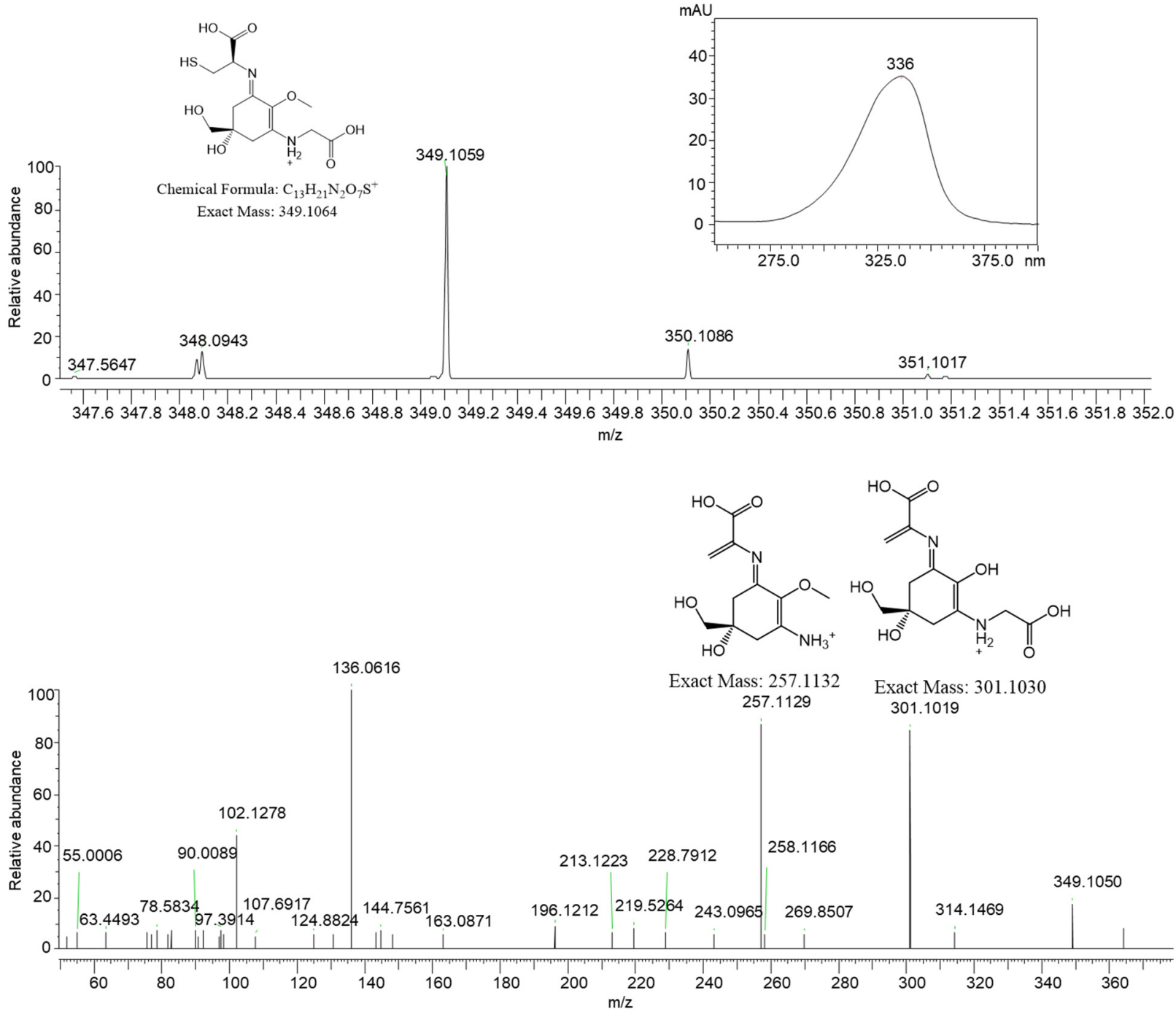

Figure S20. The maximal UV absorbance, HRMS and MS/MS spectra of MG-Cys produced in the MysD reaction. 

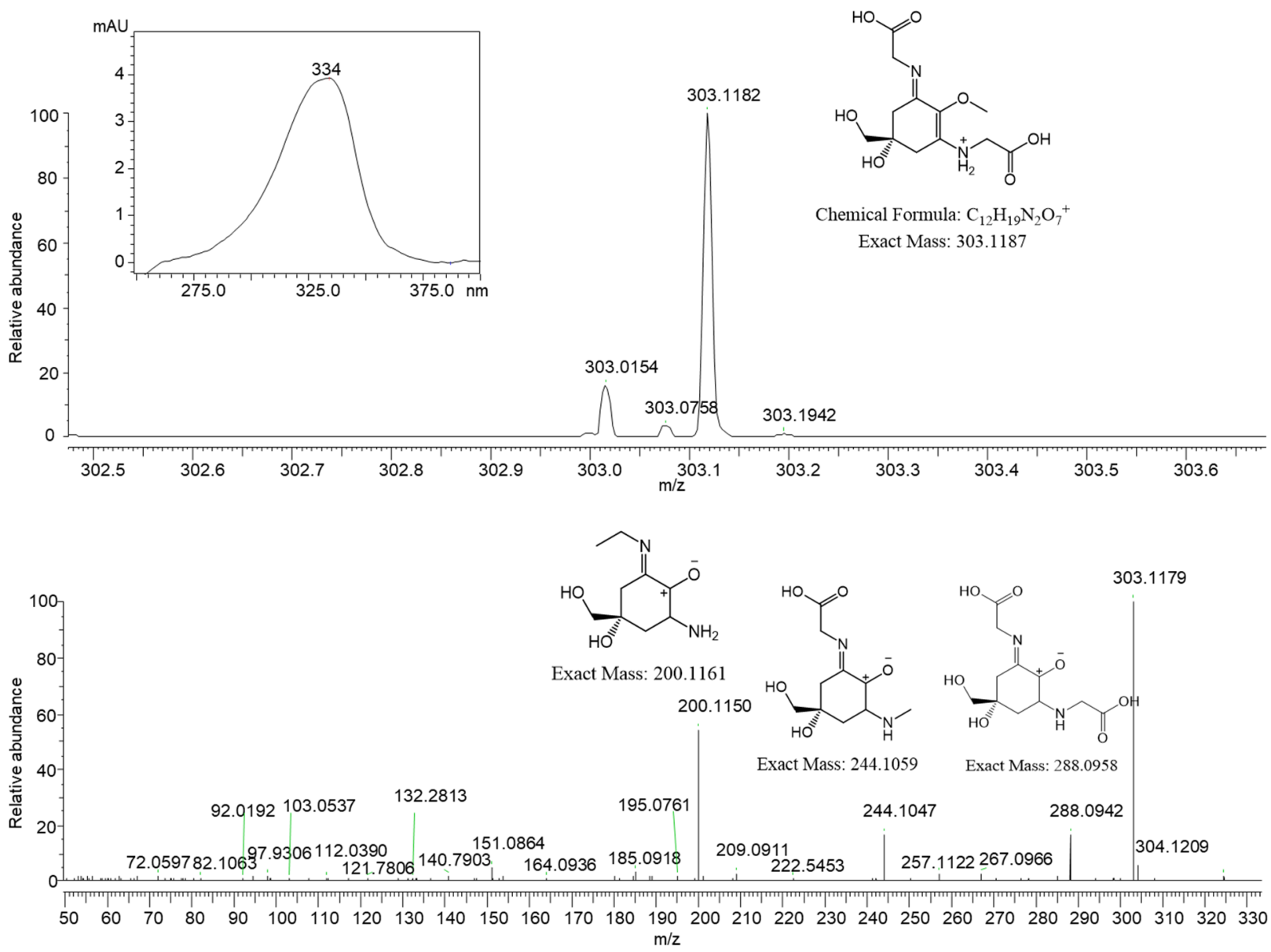

Figure S21. The maximal UV absorbance, HRMS and MS/MS spectra of mycosporine-2-Gly produced in the MysD reaction.

\section{References:}

(1) Orfanoudaki, M.; Hartmann, A.; Ngoc, H. N.; Gelbrich, T.; West, J.; Karsten, U.; Ganzera, M., Mycosporine-like amino acids, brominated and sulphated phenols: Suitable chemotaxonomic markers for the reassessment of classification of Bostrychia calliptera (Ceramiales, Rhodophyta). Phytochemistry 2020, 174, 112344. doi: 10.1016/j.phytochem.2020.

(2) Zallot, R.; Oberg, N.; Gerlt, J. A., The EFI web resource for genomic enzymology tools: Leveraging protein, genome, and metagenome databases to discover novel enzymes and metabolic pathways. Biochemistry 2019, 58 (41), 4169-4182. 